\title{
EQUIVARIANT LAGRANGIAN FLOER COHOMOLOGY VIA SEMI-GLOBAL KURANISHI STRUCTURES
}

\author{
ERKAO BAO AND KO HONDA
}

\begin{abstract}
Using a simplified version of Kuranishi perturbation theory that we call semi-global Kuranishi structures, we give a definition of the equivariant Lagrangian Floer cohomology of a pair of Lagrangian submanifolds that are fixed under a finite symplectic group action and satisfy certain simplifying assumptions.
\end{abstract}

\section{CONTENTS}

1. Introduction

2. Equivariant semi-global Kuranishi structure

3. Orientations

4. Equivariance of curve counting

5. Equivariant Lagrangian Floer cohomology

References

\section{INTRODUCTION}

Let $G$ be a finite group. The equivariant Lagrangian Floer cohomology for a pair of Lagrangians fixed under a symplectic $G$-action was first defined and studied in $[\overline{\mathrm{KS}}]$ and later in $[\mathrm{SS}, \mathrm{He} 1, \overline{\mathrm{He}} 2, \mathrm{He} 3, \overline{\mathrm{HLS}}]$. One of the main difficulties in defining such a theory is achieving transversality of the moduli spaces of $J$-holomorphic curves using an equivariant almost complex structure $J$. Indeed, there are obstructions to the existence of equivariant regular almost complex structures; see [KS, SS]. The paper [HLS] uses an infinite family of non-equivariant regular almost complex structures and an algebraic approach to define equivariant cohomology.

The goal of this paper is to give an alternate definition of equivariant Lagrangian Floer cohomology using an equivariant almost complex structure $J$ that is not necessarily regular. This involves constructing an equivariant version of a semi-global Kuranishi structure, which is a simplified version of the Kuranishi structures of [FOn, $\overline{F O 3]}$ used in [BH2]; compare to [MW] for the Kuranishi atlas formulation.

Date: This version: April 22, 2020.

2000 Mathematics Subject Classification. Primary 57M50; Secondary 53D10,53D40.

Key words and phrases. symplectic structure, Floer homology. 
It is worth mentioning that there is a construction of equivariant Kuranishi charts in $[\mathrm{Fu}]$ in a more general situation via a quite different approach.

Let $(M, \omega)$ be a compact symplectic manifold of dimension $2 n$, and let $L_{0}$ and $L_{1}$ be oriented Lagrangian submanifolds of $M$ that intersect transversely. Suppose $G$ acts on $(M, \omega)$ symplectically and satisfies $g\left(L_{i}\right)=L_{i}$ for all $g \in G$ and $i=0,1$; and that $G$ fixes the orientations of $L_{i}$.

We make the following simplifying assumption:

(S) the maps $\pi_{2}(M) \stackrel{\int \omega}{\rightarrow} \mathbb{R}$ and $\pi_{2}\left(M, L_{i}\right) \stackrel{\int \omega}{\rightarrow} \mathbb{R}$ for $i=0,1$ have image 0 .

More informally, $[\mathbf{( S )}]$ says that for all almost complex structures we consider we want to avoid disk and sphere bubbles.

We also assume that either $M$ is closed or $M$ has contact type boundary, i.e., on a neighborhood of $\partial M$ there exists a 1-form $\sigma$ such that $\omega=d \sigma$ and the vector field $X$ defined by $\iota_{X} \omega=\sigma$ is positively transverse to $\partial M$. Note that because $\omega$ is $G$-invariant, by averaging $\sigma$ over $G$, we can take $\sigma$ to be $G$-invariant.

The time- $r$ flow $\phi^{r}$ of $X$ gives a diffeomorphism $\Phi$ from $(-\epsilon, 0] \times \partial M$ to a neighborhood of $\partial M$ defined by $(r, m) \mapsto \phi^{r}(m)$. Since

$$
\mathcal{L}_{X} \sigma=d \iota_{X} \sigma+\iota_{X} d \sigma=d \iota_{X} \iota_{X} \omega+\iota_{X} \omega=\sigma,
$$

we have $\left(\phi^{r}\right)^{*} \sigma=e^{r} \sigma$. Setting $\alpha=\left.\sigma\right|_{\partial M}$, we obtain $\Phi^{*} \sigma=e^{r} \alpha$. Since $\alpha \wedge$ $(d \alpha)^{n-1}=\left.\left(\iota_{X} \omega^{n}\right)\right|_{\partial M}$ and $X$ is transverse to $\partial M, \alpha \wedge(d \alpha)^{n-1}$ is a volume form on $\partial M$, and hence $\alpha$ is a contact form. We denote by $\xi=\operatorname{ker} \alpha$ the contact structure and $R_{\alpha}$ the Reeb vector field of $\alpha$.

Let $J$ be a $G$-invariant, $\omega$-compatible almost complex structure on $M$, i.e., $\omega(\cdot, J \cdot)$ is a $G$-invariant Riemannian metric. Near $\partial M$ we assume that $J$ is convex at the boundary. More specifically:

(J) on the collar neighborhood $\left((-\epsilon, 0] \times \partial M, e^{r} \alpha\right), J$ is compatible with $d \alpha$ and maps $\xi$ to $\xi$ and $\partial_{r}$ to the Reeb vector field $R_{\alpha}$ of $\alpha$.

Exact case. One special case for which (S) holds is:

- $(M, \omega=d \sigma)$ is a Liouville domain, i.e., $M$ is compact and the Liouville vector field $X$ defined by $\iota_{X} \omega=\sigma$ points out of $\partial M$;

- $L_{0}$ and $L_{1}$ are compact exact Lagrangians in $M$ with Legendrian boundary, where exactness means that $\left.\sigma\right|_{L_{i}}$ is an exact 1 -form on $L_{i}$ for $i=0,1$.

To orient the relevant moduli spaces of $J$-holomorphic strips, following [FO3, Section 8.1] we assume that:

(O) the pair $\left(L_{0}, L_{1}\right)$ is equipped with a relative spin structure which is preserved by $G$.

See Section 3 for more details on the auxiliary orientation data including relative spin structures, and Section 3.6 for the notion of a $G$-invariant relative spin structure. In particular we assume that $L_{0}$ and $L_{1}$ are oriented. (See Seidel [Se] for orientations using Pin structures and Solomon [So] for orientations using relative Pin structures.) 
We denote by $R$ the Novikov ring

$$
\left\{\sum_{i=0}^{\infty} a_{i} T^{\lambda_{i}} \mid a_{i} \in \mathbb{Z}, \lambda_{i} \in \mathbb{R}^{\geq 0}, \lambda_{0}=0 \text { and } \lim _{i \rightarrow \infty} \lambda_{i}=\infty\right\},
$$

where $T$ is a formal parameter.

The Lagrangian Floer cochain complex $C F^{\bullet}\left(L_{0}, L_{1}\right)$ of the pair $\left(L_{0}, L_{1}\right)$ is the free module over the coefficient ring $R$ generated by $L_{0} \cap L_{1}$ with differential $d$ whose definition we give below.

For $p, q \in L_{0} \cap L_{1}$, let $\pi_{2}(p, q)$ be the set of homotopy classes of continuous maps $u:[0,1] \times[0,1] \rightarrow M$ with boundary conditions $u(0, t)=q, u(1, t)=p$, $u(s, 0) \in L_{0}, u(s, 1) \in L_{1}$. Let $\widetilde{\mathcal{M}}_{J}(p, q ; A)$, where $A \in \pi_{2}(p, q)$, be the space of smooth maps $u: \mathbb{R} \times[0,1] \rightarrow M$ that satisfy:

(A1) $\bar{\partial}_{J} u:=u_{s}+J(u) u_{t}=0$;

(A2) $\left.u\right|_{\mathbb{R} \times\{i\}} \subseteq L_{i}$ for $i \in\{0,1\}$;

(A3) $\lim _{s \rightarrow-\infty} u(s, t)=q$ and $\lim _{s \rightarrow+\infty} u(s, t)=p$; and

(A4) $[u]=A$.

Note that $\mathbb{R}$ acts on $\widetilde{\mathcal{M}}_{J}(p, q ; A)$ by translation in the domain and we denote

$$
\mathcal{M}_{J}(p, q ; A):=\widetilde{\mathcal{M}}_{J}(p, q ; A) / \mathbb{R} .
$$

We also denote the virtual (= expected) dimension of $\mathcal{M}_{J}(p, q ; A)$ by $\operatorname{vim} \mathcal{M}_{J}(p, q ; A)$.

Notation 1.0.1. We use the notation $\overline{\mathcal{M}}_{J}(p, q ; A)$ to mean the space of possibly broken strips from $p$ to $q$ in the class $A$ and

$$
\partial \mathcal{M}_{J}(p, q ; A)=\overline{\mathcal{M}}_{J}(p, q ; A)-\mathcal{M}_{J}(p, q ; A) .
$$

Suppose for the moment that $J$ is regular. Then we have the differential

$$
\begin{aligned}
& d: C F^{\bullet}\left(L_{0}, L_{1}\right) \rightarrow C F^{\bullet}\left(L_{0}, L_{1}\right) \\
& d[p]=\sum_{q \in L_{0} \cap L_{1}, A \in \pi_{2}(p, q)} \# \mathcal{M}_{J}(p, q ; A) \cdot T^{\int_{A} \omega}[q],
\end{aligned}
$$

where $\# \mathcal{M}_{J}(p, q ; A)=0$ if $\operatorname{vdim} \mathcal{M}_{J}(p, q ; A) \neq 0$. Note that for each $\lambda \geq 0$, the number of $(q, A)$ such that $\omega(A) \leq \lambda$ and $\mathcal{M}_{J}(p, q ; A) \neq \emptyset$ is finite. Then as usual one shows that $d^{2}=0$ and defines the usual Lagrangian Floer homology by $H F^{\bullet}\left(L_{0}, L_{1}\right):=\operatorname{ker} d / \operatorname{Im} d$.

We recall the definition of equivariant cohomology of a space $Y$ with a $G$-action. Let $B G$ be the classifying space of $G$ and let $E G$ be the universal bundle over $B G$. The diagonal action of $G$ on $E G \times Y$ is free and the quotient is denoted by $E G \times{ }_{G} Y$. The $G$-equivariant cohomology of $Y$ with coefficient ring $R$ is defined to be $H^{\bullet}\left(E G \times_{G} Y ; R\right)$. Let $C_{\bullet}(A)$ be the singular chain complex of the space $A$ over $R$ and $C^{\bullet}(A)=\operatorname{Hom}_{R}(C \bullet(A), R)$ be the singular cochain complex of $A$. Since the singular chain complexes and cochain complexes of $E G$ and $Y$ are invariant under the $G$-action and their boundary maps are $G$-equivariant, they can 
be viewed as complexes over the group ring $R[G]$. Then we have

$$
\begin{aligned}
H^{k}\left(E G \times_{G} Y ; R\right) & \cong H^{k}\left(\operatorname{Hom}_{R}\left(C \bullet\left(E G \times_{G} Y\right), R\right)\right) \\
& \cong H^{k}\left(\operatorname{Hom}_{R}\left(C \bullet(E G) \otimes_{R[G]} C \bullet(Y), R\right)\right) \\
& \cong H^{k}\left(\operatorname{Hom}_{R[G]}\left(C \bullet(E G), C^{\bullet}(Y)\right)\right) .
\end{aligned}
$$

In the second and third lines we are taking the $k$-th cohomology of the total complex of a double complex. We are also viewing $C_{\bullet}(E G)$ as a complex of right $R[G]$-modules and $C_{\bullet}(Y)$ as a complex of left $R[G]$-modules. We can also take a smaller model for $C_{\bullet}(E G)$ : The projective resolution $P_{\bullet}$ of $R$ over $R[G]$ is chain homotopic to $C_{\bullet}(E G)$ and

$$
H^{k}\left(E G \times_{G} Y ; R\right) \cong H^{k}\left(\operatorname{Hom}_{R[G]}\left(P_{\bullet}, C^{\bullet}(Y)\right)\right) .
$$

Returning to the "usual" definition of equivariant Lagrangian Floer cohomology assuming $J$ is regular, we replace $C^{\bullet}(Y)$ by $C F^{\bullet}\left(L_{0}, L_{1}\right)$ in Equation (1.0.1). More precisely, since $J$ is invariant under the $G$-action, we have

$$
\# \mathcal{M}_{J}(p, q ; A)=\# \mathcal{M}_{J}(g(p), g(q) ; g(A))
$$

for all $g \in G$. Hence $d$ is a $R[G]$-linear map on $C F^{\bullet}\left(L_{0}, L_{1}\right)$. We can then define the $G$-equivariant Lagrangian Floer cohomology group $H F_{G}^{\bullet}\left(L_{0}, L_{1}\right)$ as the cohomology of the total complex of $\operatorname{Hom}_{R[G]}\left(P_{\bullet}, C F^{\bullet}\left(L_{0}, L_{1}\right)\right)$.

Example 1.0.2. [F] Let $f: L_{0} \rightarrow \mathbb{R}$ be a $G$-equivariant Morse function and let $L_{1}$ be $\operatorname{graph}(\epsilon \cdot d f) \subset T^{*} L_{0}$ for some small $\epsilon>0$. Then $H F_{G}^{\bullet}\left(L_{0}, L_{1}\right) \cong H_{G}^{\bullet}\left(L_{0} ; R\right)$.

In general, a $G$-invariant $J$ is not regular and the moduli space $\mathcal{M}_{J}(p, q ; A)$ is not transversely cut out. The main contribution of this paper is to obtain a $G$ equivariant cochain complex $C F^{\bullet}\left(L_{0}, L_{1}\right)$ when $J$ is not regular by constructing an equivariant version of a semi-global Kuranishi structure, initially developed in [BH2] for contact homology. The equivariant semi-global Kuranishi structure comes with a section $\mathfrak{s}$, and while the Kuranishi structure itself is $G$-equivariant, the section is not. This creates some difficulties, but interestingly enough there is a perturbed count of $\# \mathcal{M}_{J}(p, q ; A)$ that still remains $G$-invariant (cf. Theorem 4.1.2).

Our main theorem is therefore the following:

Theorem 1.0.3 (Equivariant Lagrangian Floer cohomology). Suppose $G$ acts on $(M, \omega)$ symplectically and for each $i=0,1, L_{i}$ is oriented, $g\left(L_{i}\right)=L_{i}$, for each $g \in G$, and $G$ fixes the orientation of $L_{i}$. If $(\boldsymbol{S})$ and $(\boldsymbol{O})$ hold, then there exists an $R$-module $H F_{G}^{\bullet}\left(L_{0}, L_{1}\right)$ which is an invariant of $\left(L_{0}, L_{1}\right)$ under $G$-equivariant Hamiltonian isotopy. Moreover, when there exists a regular $G$-invariant $\omega$-compatible almost complex structure on $M$ satisfying $(J)$ the usual definition of equivariant Lagrangian Floer cohomology can be made and agrees with $H_{G}^{\bullet}\left(L_{0}, L_{1}\right)$.

If we want to equip the Lagrangian Floer homology groups with a $\mathbb{Z}$-grading, we assume that $\left(L_{0}, L_{1}\right)$ is a $G$-equivariant graded Lagrangian pair; see Section 5.1 for details. 
The definition of $H F_{G}^{\bullet}\left(L_{0}, L_{1}\right)$ is given in Section 5.2 and its invariance under $G$-equivariant Hamiltonian isotopy is given in Section 5.4. Most of the work is devoted to the construction of the semi-global Kuranishi structure in Section 2 and the equivariance of the curve count in Section 4 . The agreement with the usual definition for regular $J$ is automatic.

Acknowledgements. We thank Kristen Hendricks, Robert Lipshitz, and Sucharit Sarkar for explaining to us their approach to equivariant Lagrangian Floer cohomology in [HLS]. The first author thanks Garrett Alston and Cecilia Karlsson for discussions on orientations and Vincent Colin for providing him a great visiting opportunity at the Lebesgue Center of Mathematics and the Université de Nantes, where part of this work was carried out. The first author also thanks the Simons Center for Geometry and Physics, where he worked on this paper.

\section{EQUIVARIANT SEMI-GLOBAL KURANISHI STRUCTURE}

The construction of the equivariant semi-global Kuranishi structure follows the same steps as that of [BH2]. The only differences are that (i) we consider sections, not multisections, and (ii) we pay attention to $G$-equivariance.

2.1. $G$-invariant almost complex structure. The following lemma is well-known.

Lemma 2.1.1. There exists an almost complex structure $J$ which is $\omega$-compatible, $G$-invariant, and satisfies $(\mathrm{J})$ if $\partial M \neq \varnothing$.

Proof. If $\partial M \neq \varnothing$, then on the collar neighborhood $U=(-\epsilon, 0] \times \partial M, \partial_{r}, R_{\alpha}$, and $\xi$ are preserved by $G$. Choose a Riemannian metric $\hat{g}$ on $M$ such that

$(\star) \partial_{r}, R_{\alpha}$, and $\xi$ are mutually orthogonal on $U$ and $\partial_{r}$ and $R_{\alpha}$ have length $e^{r / 2}$.

Let $g$ be the average of $\hat{g}$ under the group action $G$. Then $g$ is preserved by $G$ and $(\star)$ holds.

From $\omega$ and $g$, we obtain the canonical $\omega$-compatible almost complex structure $J$ on $M$ by the usual polar decomposition argument; see for example [Si, Proposition 12.3] and [MS1, Proposition 2.50]. More precisely, we define $A: T M \rightarrow T M$ by $\omega(u, v)=g(A u, v)$ and the almost complex structure $J$ by $J=\left(\sqrt{A^{*} A}\right)^{-1} A$, where $A^{*}$ is the $g$-adjoint of $A$. It is not hard to check that $J$ is $\omega$-compatible and $G$-invariant and that $J$ maps $\partial_{r} \mapsto R_{\alpha}$ and $J \xi=\xi$. Hence $(\mathbf{J})$ is satisfied if $\partial M \neq \varnothing$.

Lemma 2.1.2. Given almost complex structures $J^{0}$ and $J^{1}$ that are $\omega$-compatible, $G$-invariant, and satisfy $(\mathrm{J})$ if $\partial M \neq \varnothing$, there exists a 1-parameter family of almost complex structures $\left\{J^{\tau}\right\}_{\tau \in[0,1]}$ connecting $J^{0}$ and $J^{1}$ such that for each $\tau \in[0,1]$, $J^{\tau}$ is $\omega$-compatible, $G$-invariant, and satisfies $(\boldsymbol{J})$ if $\partial M \neq \varnothing$.

Proof. Define the metrics $g^{i}(\cdot, \cdot):=\omega\left(\cdot, J^{i} \cdot\right)$ for $i \in\{0,1\}$. We can connect $g^{0}$ and $g^{1}$ by a 1-parameter family of $G$-invariant metrics $\left\{g^{\tau}\right\}_{\tau \in[0,1]}$. It is not hard to see that we can take the $g^{\tau}$ so that $(\star)$ holds for each $\tau \in[0,1]$. Then we can define $\left\{J^{\tau}\right\}_{\tau \in[0,1]}$ as in the proof of Lemma 2.1.1 
From now on we assume $\omega, J$, and $g$ are compatible and $G$-invariant. It is easy to check that we can further choose $J$ such that for any $p \in L_{0} \cap L_{1}, J\left(T_{p} L_{0}\right)=$ $T_{p} L_{1}$. In later calculations, we implicitly use an identification of $\left(T_{p} M, J\right)$ with $\left(\mathbb{R}^{n} \oplus i \mathbb{R}^{n}, i\right)$ that maps $T_{p} L_{0}$ to the $\mathbb{R}^{n}$ factor and $T_{p} L_{1}$ to the $i \mathbb{R}^{n}$ factor.

2.2. Fredholm setup. Let $S=\mathbb{R} \times[0,1]$ with coordinates $(s, t)$ and the standard complex structure $j$ which maps $\partial_{s} \mapsto \partial_{t}$. Let $p, q \in L_{0} \cap L_{1}[1]$

For $k \geq 2$, let $\mathcal{B}^{k+1, p}=\mathcal{B}^{k+1, p}(p, q ; A)$ be the space of maps $u: S \rightarrow M$ in $W^{k+1, p}(S, M)$ satisfying (A2) (A4) and such that there exist $\rho_{+}, \rho_{-} \in \mathbb{R}, \xi_{+} \in$ $W^{k+1, p}\left(S, T_{p} M\right)$, and $\xi_{-} \in W^{k+1, p}\left(S, T_{q} M\right)$ for which

- $u(s, t)=\exp _{p} \xi_{+}(s, t)$ for $s \geq \rho_{+}$,

- $u(s, t)=\exp _{q} \xi_{-}(s, t)$ for $s \leq \rho_{-}$.

Here the exponential map exp is taken with respect to the $G$-invariant $g$. Let

$$
\pi: \mathcal{E}^{k, p}=\mathcal{E}^{k, p}(p, q ; A) \rightarrow \mathcal{B}^{k+1, p}
$$

be the smooth Banach bundle with fiber

$$
\mathcal{E}_{u}^{k, p}=\pi^{-1}(u)=W^{k, p}\left(S, \wedge^{0,1} S \otimes_{J} u^{*} T M\right) .
$$

Then

$$
\bar{\partial}_{J}: \mathcal{B}^{k+1, p} \rightarrow \mathcal{E}^{k, p}, \quad u \mapsto\left(u_{s}+J(u) u_{t}\right) \otimes_{J}(d s-i d t)
$$

is a Fredholm section and $\bar{\partial}_{J}^{-1}(0)=\widetilde{\mathcal{M}}_{J}(p, q)$.

Let $\nabla$ be the Levi-Civita connection on $M$ with respect to $g$. Let $D_{u}$ be the differential

$$
\left(\bar{\partial}_{J}\right)_{*}: T_{u} \mathcal{B}^{k+1, p} \rightarrow T_{\left(u, \bar{\partial}_{J} u\right)} \mathcal{E}^{k, p}
$$

postcomposed with the projection to $\mathcal{E}_{\left(u, \bar{\partial}_{J} u\right)}^{k, p}$. Let us write $\mathcal{W}^{k+1, p}\left(S, u^{*} T M\right)$ for $\xi \in W^{k+1, p}\left(S, u^{*} T M\right)$ satisfying $\xi(s, 0) \in T_{u(s, 0)} L_{0}$ and $\xi(s, 1) \in T_{u(s, 1)} L_{1}$. Then, by [MS2, Proposition 3.1.1],

$$
D_{u}: \mathcal{W}^{k+1, p}\left(S, u^{*} T M\right) \rightarrow W^{k, p}\left(S, \wedge^{0,1} S \otimes_{J} u^{*} T M\right)
$$

is given by

$$
\begin{aligned}
D_{u} \xi & =\frac{1}{2}(\nabla \xi+J \nabla \xi \circ j)-\frac{1}{2} J\left(\nabla_{\xi} J\right) \partial_{J} u \\
& =\frac{1}{2}\left[\left(\nabla_{s} \xi+J \nabla_{t} \xi\right)-\frac{1}{2} J\left(\nabla_{\xi} J\right)\left(u_{s}-J u_{t}\right)\right] \otimes_{J}(d s-i d t) .
\end{aligned}
$$

By abuse of notation, we are not distinguishing between sections of $u^{*} T M$ and sections of $T M$ along $u$.

In what follows we will usually write $\pi: \mathcal{E} \rightarrow \mathcal{B}$. Note that, as $s \rightarrow \pm \infty$, $\left(\nabla_{s} \xi+J \nabla_{t} \xi\right) \rightarrow \partial_{s} \xi+J(p) \partial_{t} \xi$ and $u_{s}, u_{t} \rightarrow 0$. This motivates the following definition.

\footnotetext{
${ }^{1}$ We will be using $p$ for both a point in $L_{0} \cap L_{1}$ and the $L^{p}$-exponent. Hopefully this will not create any confusion.
} 


\subsection{The asymptotic operator. Consider}

$$
\mathcal{W}_{p}=\left\{\xi \in C^{\infty}\left([0,1], T_{p} M\right) \mid \xi(i) \in T_{p} L_{i}, i=0,1\right\},
$$

with inner product

$$
\left\langle\xi_{1}, \xi_{2}\right\rangle=\int_{0}^{1} g_{p}\left(\xi_{1}(t), \xi_{2}(t)\right) d t
$$

The asymptotic operator $A=A_{p}: \mathcal{W}_{p} \rightarrow \mathcal{W}_{p}$ is the self-adjoint operator

$$
A \xi(t)=-J(p) \frac{\partial}{\partial t} \xi(t) .
$$

We list the eigenvalues of $A$

$$
\cdots \leq \lambda_{-2} \leq \lambda_{-1}<0<\lambda_{1} \leq \lambda_{2} \leq \cdots
$$

with corresponding eigenfunctions

$$
\cdots, f_{-2}^{p}, f_{-1}^{p}, f_{1}^{p}, f_{2}^{p} \cdots,
$$

chosen so that the $f_{i}^{p}$ form an $L^{2}$-orthonormal basis of $\mathcal{W}_{p}$.

Model calculation for the adjoint. Consider the map $u: \mathbb{R} \times[0,1] \rightarrow \mathbb{C}$ with boundary conditions $u(s, 0) \in L_{0}=\mathbb{R}$ and $u(s, 1) \in L_{1}=i \mathbb{R}=J \mathbb{R}$ and decay conditions $\lim _{s \rightarrow \pm \infty} u(s, t)=0$. Consider the Cauchy-Riemann operator $D u=\frac{\partial u}{\partial s}+J \frac{\partial u}{\partial t}$.

We calculate the adjoint operator $D^{*} v$, for any compactly supported $v: \mathbb{R} \times$ $[0,1] \rightarrow \mathbb{C}$. It satisfies $\langle D u, v\rangle=\left\langle u, D^{*} v\right\rangle$, where $\langle$,$\rangle denotes the L^{2}$-norm. More precisely, we have

$$
\begin{aligned}
& \begin{aligned}
\int_{\mathbb{R} \times[0,1]}\left(\frac{\partial u}{\partial s}+J \frac{\partial u}{\partial t}, v\right) & d s d t=\int_{\mathbb{R} \times[0,1]}\left(\frac{\partial u}{\partial s}, v\right)+\left(J \frac{\partial u}{\partial t}, v\right) d s d t \\
= & \int_{\mathbb{R} \times[0,1]}\left(\frac{\partial}{\partial s}(u, v)-\left(u, \frac{\partial v}{\partial s}\right)+\frac{\partial}{\partial t}(J u, v)-\left(J u, \frac{\partial v}{\partial t}\right)\right) d s d t \\
& =-\int_{\mathbb{R} \times[0,1]}\left(u, \frac{\partial v}{\partial s}-J \frac{\partial v}{\partial t}\right) d s d t+\int_{\mathbb{R} \times[0,1]} \frac{\partial}{\partial t}(J u, v) d s d t
\end{aligned}
\end{aligned}
$$

Here $(\cdot, \cdot)$ is the real part of the standard Hermitian inner product on $\mathbb{C}$. Observe that:

$$
\int_{-\infty}^{\infty} \frac{\partial}{\partial s}(u, v) d s=\left.(u, v)\right|_{s=-\infty} ^{s=+\infty}=0
$$

by the decay conditions at $s= \pm \infty$. We also have

$$
\int_{0}^{1} \frac{\partial}{\partial t}(J u, v) d t=\left.(J u, v)\right|_{t=0} ^{t=1}=\left.\omega(J u, J v)\right|_{t=0} ^{t=1}=\left.\omega(u, v)\right|_{t=0} ^{t=1} .
$$

The following claim implies the adjoint is $D^{*} v=-\left(\frac{\partial v}{\partial s}-J \frac{\partial v}{\partial t}\right)$, subject to the restriction of the domain to $v$ satisfying $v(s, 0) \in L_{0}=\mathbb{R}$ and $v(s, 1) \in L_{1}=J \mathbb{R}$.

Claim 2.3.1. If $\langle D u, v\rangle=0$ for all $u$, then $v$ satisfies $D^{*} v=0$ and boundary conditions $v(s, 0) \in L_{0}=\mathbb{R}$ and $v(s, 1) \in L_{1}=J \mathbb{R}$. 
Proof. By Equations (2.3.1) and (2.3.2), if $\langle D u, v\rangle=0$ for all $u$, then

$$
\int_{\mathbb{R} \times[0,1]}\left(u, D^{*} v\right) d s d t+\int_{\mathbb{R}}((J u(s, 1), v(s, 1))-(J u(s, 0), v(s, 0))) d s=0
$$

for all $u$. We can decouple this equation into two pieces by considering $u$ that are supported in the interior of $\mathbb{R} \times[0,1]$ and on small neighborhoods of boundary points. Hence we obtain the conditions $D^{*} v=0$ and $v(s, 0) \in L_{0}=\mathbb{R}$ and $v(s, 1) \in L_{1}=J \mathbb{R}$.

2.4. Interior semi-global Kuranishi charts. Let us first consider a single moduli space

$$
\mathcal{M}_{J}=\mathcal{M}_{J}(p, q ; A)=\widetilde{\mathcal{M}}_{J}(p, q ; A) / \mathbb{R} .
$$

We will often suppress the almost complex structure $J$ from the notation when it is clear from the context. Let us also abbreviate $\widetilde{\mathcal{M}}=\widetilde{\mathcal{M}}(p, q ; A), \mathcal{B}=\mathcal{B}(p, q ; A)$, and $\mathcal{E}=\mathcal{E}(p, q ; A)$.

Definition 2.4.1. An interior semi-global Kuranishi chart is a quadruple ( $\mathbb{K}, \pi$ : $\mathbb{E} \rightarrow \mathbb{V}, \bar{\partial}, \psi)$, where:

(i) $\mathbb{K} \subset \mathcal{M}$ is a large compact subset; if $\mathcal{M}$ is compact, we take $\mathbb{K}=\mathcal{M}$;

(ii) $\pi: \mathbb{E} \rightarrow \mathbb{V}$, called the obstruction bundle, is a finite rank vector bundle over a finite-dimensional manifold;

(iii) $\bar{\partial}: \mathbb{V} \rightarrow \mathbb{E}$ is a section;

(iv) $\psi: \bar{\partial}^{-1}(0) \rightarrow \mathcal{M}$ is a homeomorphism onto an open subset of $\mathcal{M}$ and $\mathbb{K} \subset \operatorname{Im}(\psi)$;

(v) $\operatorname{dim} \mathbb{V}-\operatorname{rk} \mathbb{E}=\operatorname{vdim} \mathcal{M}$.

If the group $G$ acts on $(\mathbb{K}, \pi: \mathbb{E} \rightarrow \mathbb{V}, \bar{\partial}, \psi)$, then the Kuranishi chart is $G$ invariant.

A section $\mathfrak{s}$ of $\pi: \mathbb{E} \rightarrow \mathbb{V}$ that is transverse to $\bar{\partial}$ is an obstruction section.

Notation 2.4.2. In (iii) we are abusing notation and writing $\bar{\partial}$ for the section to indicate that it descends from $\bar{\partial}: \mathcal{B} \rightarrow \mathcal{E}$; for the charts we construct, the sections $\bar{\partial}$ are consistent with one another. We will also often abuse notation and write $\mathbb{K} \subset \mathbb{V}$ without referring to the map $\psi$.

The goal of this subsection is to construct a $G$-equivariant interior semi-global Kuranishi chart over a large $G$-invariant compact subset $\mathbb{K} \subset \mathcal{M}$.

Let $B_{q} \subset M$ be a sufficiently small disk neighborhood of $q \in L_{0} \cap L_{1}$. Given $m \in B_{q}$, let

$$
\Gamma_{m}^{q}: T_{q} B_{q} \rightarrow T_{m} B_{q}
$$

be the parallel transport with respect to the Levi-Civita connection of $g$ along the shortest geodesic from $q$ to $m$. Next we define the $t \in[0,1]$-dependent section $F_{j}^{q}:[0,1] \times B_{q} \rightarrow T B_{q}$ of $T B_{q} \rightarrow B_{q}$ by

$$
F_{j}^{q}(t, m)=\Gamma_{m}^{q}\left(f_{j}^{q}(t)\right)
$$

where $f_{j}^{q}$ are the eigenfunctions of $A_{q}$. 


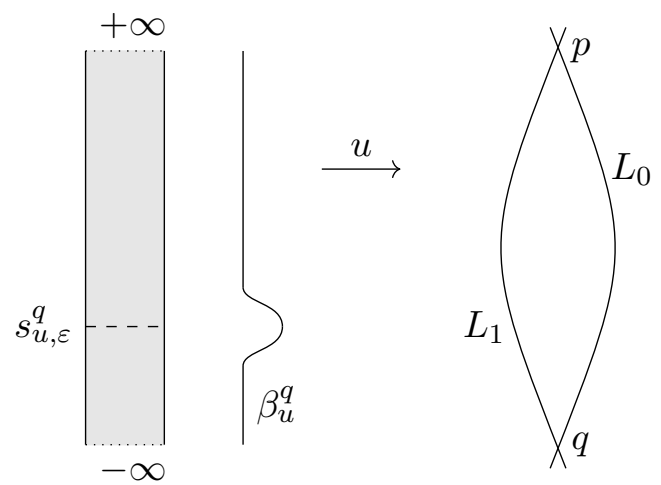

FIGURE 1.

Definition 2.4.3 (The map $a_{q}$ ). Let $\mathcal{P}\left(B_{q}\right)$ be the space of $C^{1}$-paths $\gamma:[0,1] \rightarrow$ $B_{q}$ satisfying $\gamma(i) \in L_{i}$ for $i \in\{0,1\}$. We then define a map

$$
a_{q}: \mathcal{P}\left(B_{q}\right) \rightarrow \mathbb{R}
$$

as follows: Let $v_{\gamma}:(-\infty, 0] \times[0,1] \rightarrow M$ be a $C^{1}$-map such that $v_{\gamma}(0, t)=\gamma(t)$, $v_{\gamma}(s, i) \in L_{i}$ for $i \in\{0,1\}$, and $\lim _{s \rightarrow-\infty} v_{\gamma}(s, t)=q$. Then $v_{\gamma}$ is a path in $\mathcal{P}\left(B_{q}\right)$ from the constant path at $q$ to $\gamma$. Then let

$$
a_{q}(\gamma)=\int_{(-\infty, 0] \times[0,1]} v_{\gamma}^{*} \omega
$$

Note that $a_{q}(\gamma)$ does not depend on the choice of path $v_{\gamma}$.

By the monotonicity lemma, there exists $\varepsilon>0$ such that for each nonconstant $v \in \widetilde{\mathcal{M}}$, there exists a unique value $s_{v, \varepsilon}^{q}$ of $s \in \mathbb{R}$ which satisfies the following:

(sq) the path $\gamma_{v, s}(t)=v(s, t)$ is contained in $B_{q}$ and $a_{q}\left(\gamma_{v, s}\right)=\varepsilon$.

Note that if $v^{\prime}(s, t)=v\left(s+s_{0}, t\right)$ then $s_{v^{\prime}, \varepsilon}^{q}=s_{v, \varepsilon}^{q}-s_{0}$.

We can also define $s_{u, \varepsilon}^{q}$ for $u \in \mathcal{B}$ which is $C^{1}$-close to $v$.

Let $\mathcal{U}=\mathcal{U}_{v} \subset \mathcal{B}$ be a sufficiently small open neighborhood of $v \in \widetilde{\mathcal{M}}$. Fix $\delta>0$ small. We pick a smooth bump function $\beta: \mathbb{R} \rightarrow[0,1]$ such that

(a) $\beta(s)=1$ for $s \in[-1,1]$, and

(b) $\beta(s)=0$ for $s \notin[-2,2]$.

We construct a section $\tilde{f}_{j}^{q}=\tilde{f}_{j}^{q, \delta}$ of $\left.\mathcal{E}\right|_{\mathcal{U}} \rightarrow \mathcal{U}$ as follows. For each $u \in \mathcal{U}$, we define

$$
\tilde{f}_{j}^{q}(u)=\beta_{u}^{q} \cdot u^{*} F_{j}^{q} \otimes_{\mathbb{C}}(d s-i d t) \in W^{k, p}\left(S, \Lambda^{0,1} S \otimes u^{*} T M\right),
$$

where $\beta_{u}^{q}: \mathbb{R} \rightarrow[0,1]$ is a smooth bump function of $s$ defined by

$$
\beta_{u}^{q}(s)=\beta\left(\delta^{-1}\left(s-s_{u, \varepsilon}^{q}\right)\right) .
$$


We denote by $E^{\ell}=E^{q, \ell} \rightarrow \mathcal{U}$ the vector subbundle of $\left.\mathcal{E}\right|_{\mathcal{U}}$ spanned by the sections $\tilde{f}_{-1}^{q}, \ldots, \tilde{f}_{-\ell}^{q}$. The $\mathbb{R}$-translation of $S=\mathbb{R} \times[0,1]$ induces an $\mathbb{R}$-action on $\mathcal{E} \rightarrow \mathcal{B}$ with respect to which the sections $\bar{\partial}$ and $\tilde{f}_{i}^{q}$ are equivariant. We denote by $\mathbb{E}^{\ell} \rightarrow \mathbb{U}$ the quotient of the bundle $E^{\ell} \rightarrow \mathcal{U}$ by the $\mathbb{R}$-action. We also introduce the vector space

$$
e^{\ell}=e^{q, \ell}=\mathbb{R}\left\langle f_{-1}^{q}, \ldots, f_{-\ell}^{q}\right\rangle .
$$

Proposition 2.4.4. There exist a sufficiently large $\ell$ and a sufficiently small open neighborhood $\mathcal{N}(\mathbb{K}) \subseteq \mathcal{B} / \mathbb{R}$ of $\mathbb{K}$ such that the vector bundle $\mathbb{E}^{\ell} \rightarrow \mathcal{N}(\mathbb{K})$, obtained by patching together charts of the form $\mathbb{E}^{\ell} \rightarrow \mathbb{U}$ with $\mathbb{U}=\mathbb{U}_{[v]}:=\mathcal{U}_{v} / \mathbb{R}$ and $[v] \in \mathbb{K}$, is transverse to the section $\left.\bar{\partial}\right|_{\mathcal{N}(\mathbb{K})}$ and is trivial with fibers that are canonically identified with $e^{\ell}$.

The proof is similar to that of [BH2, Theorem 5.1.2] and will be omitted. In a nutshell, this is because $D_{u}^{*}(\zeta \otimes(d s-i d t))$ is approximated by $-\left(\partial_{s} \zeta+A \zeta\right)$ for $s \ll 0$ and each nonzero element of $\operatorname{Ker} D_{u}^{*}$ has a negative end that is dominated by $e^{-\lambda_{j} s} f_{j}(t)$ for some $j<0$.

We then define

$$
\mathbb{V}:=\bar{\partial}^{-1}\left(\left.\mathbb{E}^{\ell}\right|_{\mathcal{N}(\mathbb{K})}\right) \subset \mathcal{N}(\mathbb{K})
$$

and restrict $\mathbb{E}^{\ell} \rightarrow \mathcal{N}(\mathbb{K})$ to $\mathbb{V}$. By shrinking $\mathbb{V}$ if necessary, we may assume that $\mathbb{V}$ is $G$-equivariant. This completes the construction of a $G$-equivariant interior semi-global Kuranishi chart for $\mathbb{K}$.

In view of the identification of the fibers of $\mathbb{E}^{\ell}$ with $e^{\ell}$, we will usually take an obstruction section $\mathfrak{s}$ on $\mathbb{E}^{\ell} \rightarrow \mathbb{V}$ to be a generic point in $e^{\ell}=e^{q, \ell}$ which is sufficiently close to the origin. A more specific choice of the generic point $s_{q} \in e^{q, \ell}$ will be made in Section 4.1 .

2.5. Boundary semi-global Kuranishi charts. In this subsection, we explain how to construct Kuranishi charts for curves that are close to breaking.

2.5.1. Simplest case. Let us consider the simplest situation where

$$
\mathcal{M}_{1}=\mathcal{M}\left(p, r ; A_{1}\right), \quad \mathcal{M}_{2}=\mathcal{M}\left(r, q ; A_{2}\right), \quad \mathcal{M}_{3}=\mathcal{M}\left(p, q ; A_{1}+A_{2}\right),
$$

$\partial \mathcal{M}_{3}=\mathcal{M}_{1} \times \mathcal{M}_{2}$, and $\mathcal{M}_{3}$ is $G$-invariant. Let $\mathbb{K}_{1}, \mathbb{K}_{2}, \mathbb{K}_{3}$ be compact subsets of $\mathcal{M}_{1}, \mathcal{M}_{2}, \mathcal{M}_{3}$, respectively,

$$
\left(\mathbb{K}_{1}, \mathbb{E}_{1} \rightarrow \mathbb{V}_{1}, \bar{\partial}\right), \quad\left(\mathbb{K}_{2}, \mathbb{E}_{2} \rightarrow \mathbb{V}_{2}, \bar{\partial}\right), \quad\left(\mathbb{K}_{3}, \mathbb{E}_{3} \rightarrow \mathbb{V}_{3}, \bar{\partial}\right)
$$

be the corresponding interior $G$-equivariant semi-global Kuranishi charts, and $\mathfrak{s}_{1} \in$ $e^{r, \ell}, \mathfrak{s}_{2} \in e^{q, \ell}, \mathfrak{s}_{3} \in e^{q, \ell}$ be the obstruction sections.

We will construct a boundary semi-global Kuranishi chart $\mathbb{E}_{(12)} \rightarrow \mathbb{V}_{(12)}$ over the curves of $\mathcal{M}_{3}$ that are close to breaking. Let $\sigma>0$ be small.

Definition 2.5.1 (Close to breaking). An element $u \in \mathcal{B}\left(p, q ; A_{1}+A_{2}\right)$ (resp. $\left.[u] \in \mathcal{B}\left(p, q ; A_{1}+A_{2}\right) / \mathbb{R}\right)$ is $\sigma$-close to a broken strip $\left(\left[u_{1}\right],\left[u_{2}\right]\right) \in \mathbb{V}_{1} \times \mathbb{V}_{2}$ if there exist representatives $u_{1}, u_{2}$ of $\left[u_{1}\right],\left[u_{2}\right]$ (resp. $u, u_{1}, u_{2}$ of $[u],\left[u_{1}\right],\left[u_{2}\right]$ ) such that 
- $\left.u\right|_{\left[\sigma^{-1}, \infty\right) \times[0,1]}$ is $\sigma$-close in the $C^{1}$-norm to $\left.u_{1}\right|_{\left[\sigma^{-1}, \infty\right) \times[0,1]}$,

- $\left.u\right|_{\left(-\infty,-\sigma^{-1}\right] \times[0,1]}$ is $\sigma$-close in the $C^{1}$-norm to $\left.u_{2}\right|_{\left(-\infty,-\sigma^{-1}\right] \times[0,1]}$,

- $\left.u\right|_{\left[-\sigma^{-1}, \sigma^{-1}\right] \times[0,1]},\left.u_{1}\right|_{\left(-\infty, \sigma^{-1}\right] \times[0,1]}$, and $\left.u_{2}\right|_{\left[-\sigma^{-1}, \infty\right) \times[0,1]}$ are $\sigma$-close in the $C^{1}$-norm to the constant map to the point $r$.

Let $\widetilde{\mathcal{G}}_{\sigma}\left(\mathbb{V}_{1}, \mathbb{V}_{2}\right) \subset \mathcal{B}\left(p, q ; A_{1}+A_{2}\right)$ be the subset of maps $u$ that are $\sigma$-close to some broken strip $\left(\left[u_{1}\right],\left[u_{2}\right]\right) \in \mathbb{V}_{1} \times \mathbb{V}_{2}$ and let $\mathcal{G}_{\sigma}\left(\mathbb{V}_{1}, \mathbb{V}_{2}\right):=\widetilde{\mathcal{G}}_{\sigma}\left(\mathbb{V}_{1}, \mathbb{V}_{2}\right) / \mathbb{R}$.

For each $u \in \widetilde{\mathcal{G}}_{\sigma}\left(\mathbb{V}_{1}, \mathbb{V}_{2}\right)$, there exists a unique value $s_{u, \varepsilon}^{r}$ of $s \in \mathbb{R}$ which satisfies the following:

(sr) the path $\gamma_{u, s}(t)=u(s, t)$ is contained in $B_{r}$ and $a_{r}\left(\gamma_{u, s}\right)=\varepsilon$.

Then for each $u \in \widetilde{\mathcal{G}}_{\sigma}\left(\mathbb{V}_{1}, \mathbb{V}_{2}\right)$ we define

$$
\tilde{f}_{j}^{r}(u)=\beta_{u}^{r} \cdot u^{*} F_{j}^{r} \otimes_{\mathbb{C}}(d s-i d t) \in W^{k, p}\left(S, \Lambda^{0,1} S \otimes u^{*} T M\right),
$$

where $\beta_{u}^{r}: \mathbb{R} \rightarrow[0,1]$ is the smooth bump function $\beta_{u}^{r}(s)=\beta\left(\delta^{-1}\left(s-s_{u, \varepsilon}^{r}\right)\right)$, and $\beta$ is as before.

Let $E^{\ell}\left(\mathbb{V}_{1}, \mathbb{V}_{2}\right) \rightarrow \widetilde{\mathcal{G}}_{\sigma}\left(\mathbb{V}_{1}, \mathbb{V}_{2}\right)$ be the vector subbundle of $\mathcal{E}_{\widetilde{\mathcal{G}}_{\sigma}\left(\mathbb{V}_{1}, \mathbb{V}_{2}\right)}$ spanned by the sections $\tilde{f}_{-1}^{r}, \ldots, \tilde{f}_{-\ell}^{r}$ and $\tilde{f}_{-1}^{q}, \ldots, \tilde{f}_{-\ell}^{q}$. By linear gluing (a simpler version of Theorem 2.6.1 described below) for $\sigma>0$ sufficiently small, $E^{\ell}\left(\mathbb{V}_{1}, \mathbb{V}_{2}\right) \rightarrow$ $\widetilde{\mathcal{G}}_{\sigma}\left(\mathbb{V}_{1}, \mathbb{V}_{2}\right)$ is transverse to $\bar{\partial}$. We then define

$$
V_{(1,2)}:=\bar{\partial}^{-1}\left(E^{\ell}\left(\mathbb{V}_{1}, \mathbb{V}_{2}\right)\right) \subset \widetilde{\mathcal{G}}_{\sigma}\left(\mathbb{V}_{1}, \mathbb{V}_{2}\right) .
$$

The quotient of $\left.E^{\ell}\left(\mathbb{V}_{1}, \mathbb{V}_{2}\right)\right|_{V_{(1,2)}} \rightarrow V_{(1,2)}$ by the $\mathbb{R}$-translation is denoted by:

$$
\pi_{(1,2)}: \mathbb{E}_{(1,2)}^{\ell} \rightarrow \mathbb{V}_{(1,2)}
$$

Observe that $\mathbb{E}_{(1,2)}^{\ell}$ is a trivial vector bundle whose fibers are canonically identified with $e^{r, \ell} \oplus e^{q, \ell}$.

Let us fix $\varepsilon^{\prime}$ satisfying $0<\varepsilon^{\prime} \ll \varepsilon$. Suppose $\sigma=\sigma\left(\varepsilon^{\prime}\right)>0$ is sufficiently small.

Definition 2.5.2 (Neck length). The neck length function is the function

$$
\begin{gathered}
\mathfrak{n l}: \widetilde{\mathcal{G}}_{\sigma}\left(\mathbb{V}_{1}, \mathbb{V}_{2}\right) \rightarrow \mathbb{R}^{+}, \\
u \mapsto s_{u,-\varepsilon^{\prime}}^{r}-s_{u, \varepsilon^{\prime}}^{r},
\end{gathered}
$$

where $s_{u,-\varepsilon^{\prime}}^{r}$ and $s_{u, \varepsilon^{\prime}}^{r}$ are the unique values defined as in (sr) above.

Observe that $\mathfrak{n l}: \widetilde{\mathcal{G}}_{\sigma}\left(\mathbb{V}_{1}, \mathbb{V}_{2}\right) \rightarrow \mathbb{R}^{+}$descends to $\mathfrak{n l}: \mathcal{G}_{\sigma}\left(\mathbb{V}_{1}, \mathbb{V}_{2}\right) \rightarrow \mathbb{R}^{+}$. Pick $\mathcal{L}=\mathcal{L}\left(\varepsilon^{\prime}, \sigma\right)>0$ large and $\varepsilon^{\prime \prime}>0$ small. After some modifications we may assume that:

(C) $\mathbb{V}_{3}$ and $\mathbb{V}_{(1,2)}$ cover $\mathcal{M}_{3}$

$\left(\mathrm{C}_{3}\right) \mathbb{V}_{3} \cap \mathcal{M}_{3}$ consists of $[u] \in \mathcal{M}_{3}-\mathcal{G}_{\sigma}\left(\mathbb{V}_{1}, \mathbb{V}_{2}\right)$ and $[u] \in \mathcal{G}_{\sigma}\left(\mathbb{V}_{1}, \mathbb{V}_{2}\right) \cap \mathcal{M}_{3}$ satisfying $\mathfrak{n} \mathfrak{l}([u])<\mathcal{L}$

$\left(\mathrm{C}_{(1,2)}\right) \mathbb{V}_{(1,2)} \cap \mathcal{M}_{3}$ consists of $[u] \in \mathcal{G}_{\sigma}\left(\mathbb{V}_{1}, \mathbb{V}_{2}\right) \cap \mathcal{M}_{3}$ satisfying $\mathfrak{n l}([u])>\mathcal{L}-\varepsilon^{\prime \prime}$;

(G) $G$ acts equivariantly on $\mathbb{E}_{(1,2)} \rightarrow \mathbb{V}_{(1,2)}$. 
The bundles $\mathbb{E}_{3} \rightarrow \mathbb{V}_{3}$ and $\mathbb{E}_{(1,2)} \rightarrow \mathbb{V}_{(1,2)}$ are related by the restrictioninclusion morphism: we first restrict $\mathbb{E}_{3} \rightarrow \mathbb{V}_{3}$ to

$$
\mathbb{V}_{3,(1,2)}:=\mathbb{V}_{3} \cap\left\{\mathcal{L}-\varepsilon^{\prime \prime}<\mathfrak{n l}([u])<\mathcal{L}\right\}
$$

and take the natural inclusion into $\mathbb{E}_{(1,2)} \rightarrow \mathbb{V}_{(1,2)}$, recalling that the fibers of $\mathbb{E}_{3}$ are canonically identified with $e^{q, \ell}$ and the fibers of $\mathbb{E}_{(1,2)}$ are canonically identified with $e^{r, \ell} \oplus e^{q, \ell}$.

Definition 2.5.3 (The function $\zeta$ ). Choose $0<\varepsilon^{\prime \prime \prime} \ll \varepsilon^{\prime \prime}$. Let

$$
\zeta:[0, \infty) \rightarrow[0,1]
$$

be a smooth function such that

- $\zeta\left(\left[0, \mathcal{L}+\varepsilon^{\prime \prime \prime}\right]\right)=0$,

- $\zeta\left(\left[\mathcal{L}+\varepsilon^{\prime \prime}-\varepsilon^{\prime \prime \prime}, \infty\right)\right)=1$, and

- its restriction to $\left(\mathcal{L}+\varepsilon^{\prime \prime \prime}, \mathcal{L}+\varepsilon^{\prime \prime}-\varepsilon^{\prime \prime \prime}\right)$ is a diffeomorphism onto $(0,1)$.

We then set

$$
\mathfrak{s}_{(1,2)}=\left(\zeta(\mathfrak{n l}) s_{r}, s_{q}\right) \in e^{r, \ell} \oplus e^{q, \ell} .
$$

In particular, $\mathfrak{s}_{(1,2)}=\left(s_{r}, s_{q}\right)$ on $\mathfrak{n l} \geq \mathcal{L}+\varepsilon^{\prime \prime}$ and $\mathfrak{s}_{(1,2)}=\left(0, s_{q}\right)$ on $\mathfrak{n l} \leq \mathcal{L}$. By the restriction-inclusion, $\mathfrak{s}_{(1,2)}$ is consistent with $\mathfrak{s}_{3}$.

2.5.2. Order of choice of constants. We outline the order in which the auxiliary constants are chosen.

(1) Choose $\varepsilon_{1}>0$ small such that $s_{u, \varepsilon^{\prime}}^{r}$ is defined for any $0<\varepsilon^{\prime}<\varepsilon_{1}$ and any $u \in \widetilde{\mathcal{M}}\left(p, r ; A_{1}\right)$. Then choose $\ell_{1}$ and $\mathbb{V}_{1}$.

(2) Choose $\varepsilon_{2}>0$ small such that $s_{v, \varepsilon^{\prime}}^{q}$ and $s_{w, \varepsilon^{\prime}}^{q}$ are defined for any $0<\varepsilon^{\prime}<$ $\varepsilon_{2}$ and any $v \in \widetilde{\mathcal{M}}\left(r, q ; A_{2}\right)$ and $w \in \widetilde{\mathcal{M}}\left(p, q ; A_{1}+A_{2}\right)$. Then choose $\ell_{2}$ and $\mathbb{V}_{2}$.

(3) Choose $\varepsilon^{\prime}>0$ such that $0<\varepsilon^{\prime} \ll \varepsilon_{1}$ and then $\sigma>0$ small such that $s_{w, \varepsilon^{\prime}}^{r}$ and $s_{w,-\varepsilon^{\prime}}^{r}$ are defined for any $w \in \widetilde{\mathcal{G}}_{\sigma}\left(\mathbb{V}_{1}, \mathbb{V}_{2}\right)$. Here $\sigma$ can be chosen to be independent of $\ell_{1}$ and $\ell_{2}$, but we may need to shrink $\mathbb{V}_{i}$ satisfying $\mathbb{K}_{i} \subseteq \mathbb{V}_{i}$ for $i=0,1$. This is because if $w$ is close to breaking into $\mathbb{V}_{1} \times \mathbb{V}_{2}$ and $\mathbb{V}_{1} \times \mathbb{V}_{2}$ is a sufficiently small neighborhood of $\mathbb{K}_{1} \times \mathbb{K}_{2}$, then $w$ is close to breaking into $\mathbb{K}_{1} \times \mathbb{K}_{2}$, which is $\ell_{1}, \ell_{2}$-independent. The neck length $\mathfrak{n l}(w)$ is then given by $s_{w,-\varepsilon^{\prime}}^{r}-s_{w, \varepsilon^{\prime}}^{r}$.

(4) Define $\mathbb{V}_{(1,2)} \subset \mathcal{G}_{\sigma}\left(\mathbb{V}_{1}, \mathbb{V}_{2}\right)$.

(5) Choose a compact $\mathbb{K}_{3} \subseteq \mathcal{M}\left(p, q ; A_{1}+A_{2}\right)$ such that if $[w] \in \mathcal{M}\left(p, q ; A_{1}+\right.$ $\left.A_{2}\right)-\mathbb{K}_{3}$, then $[w]$ is $\sigma$-close to breaking into $\mathbb{V}_{1} \times \mathbb{V}_{2}$, i.e., $[w] \in$ $\mathcal{G}_{\sigma}\left(\mathbb{V}_{1}, \mathbb{V}_{2}\right)$.

(6) Pick $\mathcal{L}>0$ large and $\varepsilon^{\prime \prime}>0$ small, and enlarge $\mathbb{K}_{3}$ if necessary so that

$$
\mathbb{K}_{3} \cap \mathbb{V}_{(1,2)} \supseteq\left\{[w] \in \mathcal{M}\left(p, q ; A_{1}+A_{2}\right) \mid \mathcal{L}-\varepsilon^{\prime \prime}<\mathfrak{n l}([w])<\mathcal{L}\right\} .
$$

(7) Choose $\ell \in \mathbb{N}$ such that $\left.\mathbb{E}^{\ell}\right|_{\mathbb{K}_{3}}$ is transverse to $\bar{\partial}$. 
(8) With the choice of $\ell$, we may need to increase $\ell_{2}$ so that $\ell=\ell_{2}$. By (3) this update does not affect $\sigma, \mathcal{L}, \varepsilon^{\prime \prime}$. To reduce the number of constants, we also choose to update $\ell_{1}$ so that $\ell_{1}=\ell_{2}=\ell$.

(9) Trim $\mathbb{V}_{3}$ and $\mathbb{V}_{(1,2)}$ so that $\left(\mathrm{C}_{3}\right)$ and $\left(\mathrm{C}_{(1,2)}\right)$ are satisfied.

(10) The constant $0<\varepsilon^{\prime \prime \prime} \ll \varepsilon^{\prime \prime}$ is as defined in Definition 2.5.3. Then we interpolate between the sections $\mathfrak{s}_{3}$ and $\mathfrak{s}_{(1,2)}$.

2.5.3. General case. In general, we construct boundary semi-global Kuranishi charts by induction on the energy.

Ordering the moduli spaces. We will explain how to order the moduli spaces $\mathcal{M}(p, q ; A)$. We remark that even if $\mathcal{M}(p, q ; A)=\emptyset$, we still need to construct a Kuranishi chart for $\mathcal{M}(p, q ; A)$ (i.e., include $\mathcal{M}(p, q ; A)$ in our list), if $A=$ $A_{1}+A_{2}$ for some $A_{1} \in \pi_{2}(p, r)$ and $A_{2} \in \pi_{2}(r, q)$, and $\mathcal{M}\left(p, r ; A_{1}\right) \neq \emptyset$ and $\mathcal{M}\left(r, q ; A_{2}\right) \neq \emptyset$.

We define

$$
\begin{gathered}
\mathfrak{M}=\left\{(p, q, A) \mid p, q \in L_{0} \cap L_{1} \text { and } A \in \pi_{2}(p, q)\right\}, \\
\mathfrak{M}^{1+}=\{(p, q, A) \in \mathfrak{M} \mid \mathcal{M}(p, q ; A) \neq \emptyset\}, \\
\lambda_{1}=\inf \left\{\omega(A) \mid(p, q ; A) \in \mathfrak{M}^{1+}\right\},
\end{gathered}
$$

and

$$
\mathfrak{M}^{1}=\left\{(p, q ; A) \in \mathfrak{M}^{1+} \mid \omega(A)=\lambda_{1}\right\} .
$$

Suppose that $\mathfrak{M}^{k}$ has been inductively defined for all $k<m$. We then define $\mathfrak{M}^{m+}$ to be the set of

$$
(p, q, A) \in \mathfrak{M} \backslash\left(\cup_{i=1}^{m-1} \mathfrak{M}^{i}\right)
$$

such that either

(1) $\mathcal{M}(p, q ; A) \neq \emptyset$; or

(2) There exist $r \in L_{0} \cap L_{1}, A_{1} \in \pi_{2}(p, r)$ and $A_{2} \in \pi_{2}(r, q)$ satisfying

(2a) $A=A_{1}+A_{2}$,

(2b) $\left(p, r ; A_{1}\right) \in \mathfrak{M}^{k_{1}}$ for some $k_{1}<m$, and

(2c) $\left(r, q ; A_{2}\right) \in \mathfrak{M}^{k_{2}}$ for some $k_{2}<m$.

Let

$$
\lambda_{m}=\inf \left\{\omega(A) \mid(p, q, A) \in \mathfrak{M}^{m+}\right\},
$$

and

$$
\mathfrak{M}^{m}=\left\{(p, q, A) \in \mathfrak{M}^{m+} \mid \omega(A)=\lambda_{m}\right\} .
$$

By Gromov compactness, one can see that for each $k, \mathfrak{M}^{k}$ is finite, and $\left\{\lambda_{k} \mid k \in\right.$ $\mathbb{N}\} \subset \mathbb{R}_{\geq 0}$ is nowhere dense.

We then order the elements of $\cup_{k=1}^{\infty} \mathfrak{M}^{k}$ as

$$
\left(p_{1}, q_{1}, A_{1}\right),\left(p_{2}, q_{2}, A_{2}\right), \ldots
$$

so that it is consistent with the ordering

$$
\mathfrak{M}^{1}, \mathfrak{M}^{2}, \ldots
$$

We denote $\mathcal{M}_{i}:=\mathcal{M}\left(p_{i}, q_{i} ; A_{i}\right)$. 
We choose an increasing sequence $N_{1}, N_{2}, \cdots \rightarrow \infty$ of integers and for each $j$ we construct a semi-global Kuranishi structure $\mathcal{K}^{(j)}$ using $\mathcal{M}_{1}, \ldots, \mathcal{M}_{N_{j}}$ and a section $\mathfrak{S}^{(j)}$ of $\mathcal{K}^{(j)}$. Later we will explain how to relate $\mathcal{K}^{(j)}$ and $\mathcal{K}^{(j+1)}$ and their sections.

For the moment we choose $N \gg 0$ and such that the finite set $\left\{\mathcal{M}_{1}, \ldots, \mathcal{M}_{N}\right\}$ of moduli spaces is $G$-invariant.

Define the source, target, and homotopy class maps

$$
\mathbf{s}(\mathcal{M}(p, q ; A))=p, \quad \mathbf{t}(\mathcal{M}(p, q ; A))=q, \quad \mathbf{h}(\mathcal{M}(p, q ; A))=A .
$$

Definition 2.5.4. A tuple $I=\left(i_{1}, \ldots, i_{k}\right)$ with $i_{j} \in\{1,2, \ldots, \rho\}$ is called an index tuple if $\omega\left(\mathbf{h}\left(\mathcal{M}_{i_{j}}\right)\right)>0$ for all $j$ and $\mathbf{t}\left(\mathcal{M}_{i_{j}}\right)=\mathbf{s}\left(\mathcal{M}_{i_{j+1}}\right)$ for all $j<k$.

If $k=1$, sometimes we write $i_{1}$ instead of $\left(i_{1}\right)$.

Definition 2.5.5. Let $I=\left(i_{1}, i_{2}, \ldots, i_{k}\right)$ be an index tuple.

(1) An index tuple $I^{\prime}$ is a simple contraction of $I$ if $I^{\prime}$ is obtained by replacing a consecutive pair $i_{j}, i_{j+1}$ by $i_{j}^{\prime}$ such that $\mathbf{h}\left(\mathcal{M}_{i_{j}^{\prime}}\right)=\mathbf{h}\left(\mathcal{M}_{i_{j}}\right)+\mathbf{h}\left(\mathcal{M}_{i_{j+1}}\right)$.

(2) An index tuple $I^{\prime}$ is a contraction of $I$ if $I^{\prime}$ is obtained from $I$ by a nonempty sequence of simple contractions. We write $I^{\prime}<I$.

(3) We write $c(I)$ for the index tuple $\left(i_{1}^{\prime}\right)$ such that $\left(i_{1}^{\prime}\right) \leq I$ (i.e., $\left(i_{1}^{\prime}\right)<I$ or $\left.\left(i_{1}^{\prime}\right)=I\right)$.

(4) Given $I^{\prime}=\left(i_{1}^{\prime}, \ldots, i_{k^{\prime}}^{\prime}\right)<I$, the blocks of I relative to $I^{\prime}$ are groupings

$$
\left(i_{1}, \ldots, i_{l_{1}}\right),\left(i_{l_{1}+1}, \ldots, i_{l_{2}}\right), \ldots,\left(i_{l_{k^{\prime}-1}+1}, \ldots, i_{l_{k^{\prime}}}\right)
$$

such that $c$ applied to the $j$ th block yields $i_{j}^{\prime}$. Note that the blocks are welldefined due to the requirement $\omega\left(\mathbf{h}\left(\mathcal{M}_{i_{j^{\prime}}^{\prime}}\right)\right)>0$ for all $j^{\prime} \in\left\{1,2, \ldots, k^{\prime}\right\}$

(5) Given $I^{\prime}=\left(i_{1}^{\prime}, \ldots, i_{k^{\prime}}^{\prime}\right)<I$, let

$$
\delta\left(I, I^{\prime}\right)=\left\{i_{1}, \ldots, i_{l_{1}-1}, i_{l_{1}+1}, \ldots, i_{l_{2}-1}, \ldots, i_{l_{k^{\prime}-1}+1}, \ldots, i_{l_{k^{\prime}}-1}\right\},
$$

where we are using block notation from (4).

We can organize the set of index tuples as a category $\mathcal{I}$, called the index tuple category, with objects which are index tuples and a unique morphism from $I^{\prime}$ to $I$ if $I^{\prime} \leq I$.

Let $\mathbb{K}_{i} \subseteq \mathcal{M}_{i}$ be the large compact subsets over which we construct the equivariant interior semi-global Kuranishi chart $\mathcal{C}_{i}=\left(\mathbb{K}_{i}, \mathbb{E}_{i} \rightarrow \mathbb{V}_{i}, \bar{\partial}_{i}, \psi_{i}\right)$ and the obstruction section $\mathfrak{s}_{i}$.

Let $I=\left(i_{1}, \ldots, i_{k}\right)$. The following construction of the boundary chart

$$
\left(\pi_{I}: \mathbb{E}_{I} \rightarrow \mathbb{V}_{I}, \bar{\partial}_{I}, \psi_{I}\right)
$$

is a straightforward generalization of Section 2.5.1; Let $\widetilde{\mathcal{G}}_{\sigma}\left(\mathbb{V}_{i_{1}}, \ldots, \mathbb{V}_{i_{k}}\right)$ be the set of maps $u$ that are $\sigma$-close to a broken strip in $\mathbb{V}_{i_{1}} \times \cdots \times \mathbb{V}_{i_{k}}$, defined in a manner analogous to Definition 2.5.1. For convenience we will also write $\widetilde{\mathcal{G}}_{\sigma}\left(\mathbb{V}_{i}\right)$ for the set of maps $u$ that are $\sigma$-close to a map in $\mathbb{V}_{i}$. Again we take $\mathcal{L}=\mathcal{L}\left(\varepsilon^{\prime}, \sigma\right)$ and $\varepsilon^{\prime \prime}>0$. 
Definition 2.5.6 (Neck length). Let $u \in \cup_{c\left(i_{1}, \ldots, i_{k}\right)=(i)} \widetilde{\mathcal{G}}_{\sigma}\left(\mathbb{V}_{i_{1}}, \ldots, \mathbb{V}_{i_{k}}\right)$.

(1) The neck length function satisfies

$$
\mathfrak{n l}_{\left(i_{1}^{\prime}, i_{2}^{\prime}\right)}^{\prime}(u)=s_{u,-\varepsilon^{\prime}}^{r}-s_{u, \varepsilon^{\prime}}^{r}
$$

if $u \in \widetilde{\mathcal{G}}_{\sigma}\left(\mathbb{V}_{i_{1}}, \ldots, \mathbb{V}_{i_{k}}\right),(i)<\left(i_{1}^{\prime}, i_{2}^{\prime}\right)<\left(i_{1}, \ldots, i_{k}\right)$, and $r=\mathbf{t}\left(\mathcal{M}_{i_{1}^{\prime}}\right)=$ $\mathbf{s}\left(\mathcal{M}_{i_{2}^{\prime}}\right)$.

(2) The modified neck length function satisfies

$$
\mathfrak{n l}_{\left(i_{1}^{\prime}, i_{2}^{\prime}\right)}(u)= \begin{cases}\lambda\left(\mathfrak{n l}_{\left(i_{1}^{\prime}, i_{2}^{\prime}\right)}^{\prime}(u)\right) & \text { if } \mathfrak{n l}_{\left(i_{1}^{\prime}, i_{2}^{\prime}\right)}^{\prime}(u) \text { is defined } \\ 0 & \text { otherwise, }\end{cases}
$$

where $\lambda: \mathbb{R}^{+} \rightarrow \mathbb{R}^{\geq 0}$ is a smooth function such that $\lambda(x)=x$ for $x \geq$ $\mathcal{L}-\varepsilon^{\prime \prime}, \lambda(x)=0$ for $x \leq \mathcal{L}-2 \varepsilon^{\prime \prime}$, and $\lambda^{\prime}(x)>0$ on $\left(\mathcal{L}-2 \varepsilon^{\prime \prime}, \mathcal{L}-\varepsilon^{\prime \prime}\right)$. We also write $\mathfrak{n l}_{i_{j}}(u)=\mathfrak{n l}_{\left(i_{1}^{\prime}, i_{2}^{\prime}\right)}(u)$ if $c\left(i_{1}, \ldots, i_{j}\right)=i_{1}^{\prime}$.

We then define the boundary charts $\pi_{I}: \mathbb{E}_{I} \rightarrow \mathbb{V}_{I}, I=\left(i_{1}, \ldots, i_{k}\right)$, whose fibers are canonically identified with $e^{r_{1}, \ell} \oplus \cdots \oplus e^{r_{k}, \ell}$ and such that:

$\left(\mathrm{C}_{I}\right) \mathbb{V}_{I} \cap \mathcal{M}_{c(I)}$ consists of $[u] \in \mathcal{G}_{\sigma}\left(\mathbb{V}_{i_{1}}, \ldots, \mathbb{V}_{i_{k}}\right) \cap \mathcal{M}_{c(I)}$ satisfying

(a) $\mathfrak{n l}_{i_{j}}([u])>\mathcal{L}-\varepsilon^{\prime \prime}$ for all $j<k$ and

(b) $\mathfrak{n l}_{i_{j}^{\prime \prime}}([u])<\mathcal{L}$ for all $i_{j}^{\prime \prime} \in \delta\left(I^{\prime \prime}, I\right)$ where $I<I^{\prime \prime}=\left(i_{1}^{\prime \prime}, \ldots, i_{k^{\prime \prime}}^{\prime \prime}\right)$.

The section $\bar{\partial}_{I}$ is the $\bar{\partial}$-operator restricted to $\mathbb{V}_{I}$ and $\psi_{I}: \bar{\partial}_{I}^{-1}(0) \rightarrow \mathbb{V}_{I}$ is the obvious inclusion.

Observe that $G$ acts on the set of index tuples. Let $G_{I} \subset G$ be the stabilizer of $I$. By trimming $\mathbb{V}_{I}$ if necessary, we may assume that $G_{I}$ acts on $\mathbb{E}_{I} \rightarrow \mathbb{V}_{I}$.

Next we discuss the restriction-inclusion morphism

$$
\phi_{I^{\prime}, I}:\left(\pi_{I^{\prime}}: \mathbb{E}_{I^{\prime}} \rightarrow \mathbb{V}_{I^{\prime}}\right) \rightarrow\left(\pi_{I}: \mathbb{E}_{I} \rightarrow \mathbb{V}_{I}\right),
$$

where $I^{\prime}=\left(i_{1}^{\prime}, \ldots, i_{k^{\prime}}^{\prime}\right)<I=\left(i_{1}, \ldots, i_{k}\right)$. We first restrict $\mathbb{E}_{I^{\prime}} \rightarrow \mathbb{V}_{I^{\prime}}$ to

$$
\mathbb{E}_{I^{\prime}, I}:=\left.\mathbb{E}_{I^{\prime}}\right|_{\mathbb{I}^{\prime}, I} \rightarrow \mathbb{V}_{I^{\prime}, I}:=\mathbb{V}_{I^{\prime}} \cap\left\{\mathcal{L}-\varepsilon^{\prime \prime}<\mathfrak{n l}_{i_{j}}([u])<\mathcal{L}, \forall i_{j} \in \delta\left(I, I^{\prime}\right)\right\} .
$$

We then consider the inclusion of vector bundles given by the commutative diagram

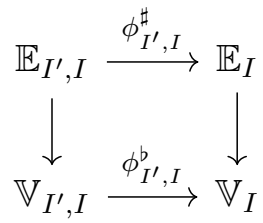

where $\phi_{I^{\prime}, I}^{b}: \mathbb{V}_{I^{\prime}, I} \rightarrow \mathbb{V}_{I}$ is the inclusion and the bundle map $\phi_{I^{\prime}, I}^{\sharp}$ is defined by canonically identifying the fibers of $\mathbb{E}_{I^{\prime}}$ and $\mathbb{E}_{I}$ with

$$
e^{r_{1}^{\prime}, \ell} \oplus \cdots \oplus e^{r_{k^{\prime}}{ }^{\prime}, \ell} \quad \text { and } \quad e^{r_{1}, \ell} \oplus \cdots \oplus e^{r_{k}, \ell},
$$

and including

$$
e^{r_{1}^{\prime}, \ell} \oplus \cdots \oplus e^{r_{k^{\prime}}^{\prime}, \ell} \subset e^{r_{1}, \ell} \oplus \cdots \oplus e^{r_{k}, \ell} .
$$

Here $r_{j}=\mathbf{t}\left(\mathcal{M}_{i_{j}}\right)$ and $r_{j}^{\prime}=\mathbf{t}\left(\mathcal{M}_{i_{j}^{\prime}}\right)$. We have 
(a) $\phi_{I^{\prime}, I}^{\sharp} \circ \bar{\partial}_{I^{\prime}}=\bar{\partial}_{I} \circ \phi_{I^{\prime}, I}^{b}$ on $\mathbb{V}_{I^{\prime}, I}$; and

(b) $\psi_{I} \circ \phi_{I^{\prime}, I}^{b}=\psi_{I^{\prime}}$ on $\bar{\partial}_{I^{\prime}}^{-1}(0) \cap \mathbb{V}_{I^{\prime}, I}$.

For $I=\left(i_{1}, \ldots, i_{k}\right)$ we set

$$
\mathfrak{s}_{I}=\left(\zeta\left(\mathfrak{n l}_{i_{1}}\right) s_{r_{1}}, \ldots, \zeta\left(\mathfrak{n l}_{i_{k-1}}\right) s_{r_{k-1}}, s_{r_{k}}\right) \in e^{r_{1}, \ell} \oplus \cdots \oplus e^{r_{k}, \ell},
$$

where the function $\zeta$ is as given in Definition 2.5.3. Denote $\mathfrak{s}_{I^{\prime}, I}:=\left.\mathfrak{s}_{I^{\prime}}\right|_{I^{\prime}, I}$. It is immediate that

$$
\phi_{I^{\prime}, I}^{\sharp} \circ \mathfrak{s}_{I^{\prime}, I}=\mathfrak{s}_{I} \circ \phi_{I^{\prime}, I}^{b}
$$

and

$$
\bar{\partial}_{I}^{-1}\left(\mathfrak{s}_{I}\right) \cap \phi_{I^{\prime}, I}^{b}\left(\mathbb{V}_{I^{\prime}, I}\right)=\phi_{I^{\prime}, I}^{b}\left(\bar{\partial}_{I^{\prime}}^{-1}\left(\mathfrak{s}_{I^{\prime}, I}\right)\right) .
$$

2.6. Gluing. The following gluing results can be proven in a manner similar to Theorems 6.4.1, 6.4.2 in [BH2] (in the contact case) and Theorem A.21 in [ES].

Theorem 2.6.1 (Gluing). For sufficiently large $R>0$, there exists a gluing map

$$
\mathfrak{G}_{\left(i_{1}, \ldots, i_{m}\right)}: \mathbb{V}_{i_{1}} \times \cdots \times \mathbb{V}_{i_{m}} \times(R, \infty)^{m-1} \rightarrow \mathbb{V}_{\left(i_{1}, \ldots, i_{m}\right)}
$$

which satisfies the following: Writing $T_{1}, \ldots, T_{m-1}$ for the coordinates on $(R, \infty)^{m-1}$,

(1) $\mathfrak{G}_{\left(i_{1}, \ldots, i_{m}\right)}$ is a $C^{1}$-diffeomorphism onto its image;

(2) $\operatorname{Im} \mathfrak{G}_{\left(i_{1}, \ldots, i_{m}\right)} \supset \mathbb{V}_{\left(i_{1}, \ldots, i_{m}\right)} \cap\left\{\mathfrak{n l}_{i_{j}} \geq R+\varepsilon^{\prime \prime}, \forall j<m\right\}$;

(3) $\mathfrak{G}_{\left(i_{1}, \ldots, i_{m}\right)}\left(\left[u_{i_{1}}\right], \ldots,\left[u_{i_{m}}\right], T_{1}, \ldots, T_{m-1}\right)$ is $\sigma$-close to the broken strip $\left(\left[u_{i_{1}}\right], \ldots,\left[u_{i_{m}}\right]\right)$ for some $\sigma>0$ (in the $C^{1}$-topology; see Definition 2.5.1);

(4) for $j=1, \ldots, m-1$, the functions $\left(\mathfrak{G}_{\left(i_{1}, \ldots, i_{m}\right)}\right)_{*} T_{j}$ and $\mathfrak{n l}_{i_{j}}$ are $C^{1}$-close;

(5) $\bar{\partial}\left(\mathfrak{G}_{\left(i_{1}, \ldots, i_{m}\right)}\left(\left[u_{i_{1}}\right], \ldots,\left[u_{i_{m}}\right], T_{1}, \ldots, T_{m-1}\right)\right)$ and $\left(\bar{\partial} u_{i_{1}}, \ldots, \bar{\partial} u_{i_{m}}\right)$, viewed as elements of $e^{r_{1}, \ell} \oplus \cdots \oplus e^{r_{k}, \ell}, r_{j}=\mathbf{t}\left(\mathcal{M}_{i_{j}}\right)$, are $C^{0}$-close;

(6) the errors in (3), (4), and (5) go to zero as all $T_{j} \rightarrow \infty$.

Theorem 2.6.2 (Iterated gluing). For sufficiently large $R>0$, there is a gluing map

$$
\begin{aligned}
\mathfrak{G}_{\left.\left(i_{1}, \ldots, i_{a}, \ldots, i_{b}\right), \ldots i_{m}\right)} & : \mathbb{V}_{i_{1}} \times \cdots \times \mathbb{V}_{i_{a}-1} \times \mathbb{V}_{\left(i_{a}, \ldots, i_{b}\right)} \\
& \times \mathbb{V}_{i_{b}+1} \times \cdots \times \mathbb{V}_{i_{m}} \times(R, \infty)^{m-(b-a)-1} \rightarrow \mathbb{V}_{\left(i_{1}, \ldots, i_{m}\right)},
\end{aligned}
$$

satisfying properties analogous to those of Theorem 2.6.1 and such that

$$
\mathfrak{G}_{\left(i_{1}, \ldots, i_{m}\right)} \text { and } \mathfrak{G}_{\left(i_{1}, \ldots,\left(i_{a}, \ldots, i_{b}\right), \ldots i_{m}\right)} \circ\left(\mathrm{id}, \ldots, \mathfrak{G}_{\left(i_{a}, \ldots, i_{b}\right)}, \ldots, \mathrm{id}\right)
$$

are $C^{1}$-close with error $\rightarrow 0$ as all the coordinates of $(R, \infty)^{m-(b-a)-1}$ go to $\infty$.

2.7. Equivariant semi-global Kuranishi structures. The Kuranishi charts constructed in Section 2.4 and 2.5 can be organized into a $G$-invariant semi-global Kuranishi structure.

Again, for the moment we work with the $G$-invariant finite set $\left\{\mathcal{M}_{1}, \ldots, \mathcal{M}_{N}\right\}$ of moduli spaces.

Our definition is similar to McDuff-Wehrheim's treatment of Kuranishi structures (called atlases) in [MW]. (1)-(3) are general properties of Kuranishi structures/atlases and (4) and (5) are specific "semi-global" properties. 
Definition 2.7.1 (Semi-global Kuranishi structure). A semi-global Kuranishi structure $\mathscr{K}$ is a category consisting of the following data:

(1) The objects are semi-global Kuranishi charts $\mathcal{C}_{I}=\left(\pi_{I}: \mathbb{E}_{I} \rightarrow \mathbb{V}_{I}, \bar{\partial}_{I}, \psi_{I}\right)$ :

(a) for each $i, C_{i}=\left(\pi_{i}: \mathbb{E}_{i} \rightarrow \mathbb{V}_{i}, \bar{\partial}_{i}, \psi_{i}\right)$ is an interior Kuranishi chart for $\mathbb{K}_{i} \subset \mathcal{M}_{i}$;

(b) for each $I=\left(i_{1}, \ldots, i_{m}\right), \pi_{I}: \mathbb{E}_{I} \rightarrow \mathbb{V}_{I}$ is a finite rank vector bundle over a finite-dimensional manifold, $\bar{\partial}_{I}: \mathbb{V}_{I} \rightarrow \mathbb{E}_{I}$ is a section, $\psi_{I}: \bar{\partial}_{I}^{-1}(0) \rightarrow \mathcal{M}_{c(I)}$ is a homeomorphism onto an open subset of $\mathcal{M}_{c(I)}$, and $\operatorname{dim} \mathbb{V}_{I}-\operatorname{rk} \mathbb{E}_{I}=\operatorname{vdim} \mathcal{M}_{c(I)} ;$ and

(c) for each $i, \cup_{c(I)=(i)} \operatorname{Im}\left(\psi_{I}\right)=\mathcal{M}_{i}$.

(2) For each $I^{\prime} \leq I$ there is a specified morphism $\phi_{I^{\prime}, I}: \mathcal{C}_{I^{\prime}} \rightarrow \mathcal{C}_{I}$ encoded by the data $\left(\mathbb{V}_{I^{\prime}, I}, \phi_{I^{\prime}, I}^{\sharp}, \phi_{I^{\prime}, I}^{b}\right)$ and given by restriction-inclusion: first restrict $\mathbb{E}_{I^{\prime}} \rightarrow \mathbb{V}_{I^{\prime}}$ to an open subset $\mathbb{V}_{I^{\prime}, I} \subset \mathbb{V}_{I^{\prime}}$ and then take the inclusion of vector bundles given by a commutative diagram

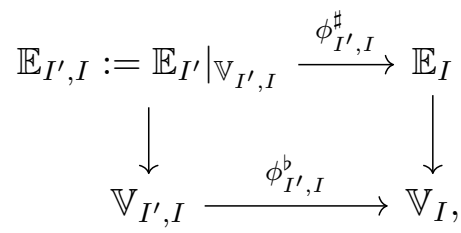

subject to:

(a) $\phi_{I^{\prime}, I}^{\sharp} \circ \bar{\partial}_{I^{\prime}}=\bar{\partial}_{I} \circ \phi_{I^{\prime}, I}^{b}$ on $\mathbb{V}_{I^{\prime}, I}$;

(b) $\psi_{I} \circ \phi_{I^{\prime}, I}^{b}=\psi_{I^{\prime}}$ on $\bar{\partial}_{I^{\prime}}^{-1}(0) \cap \mathbb{V}_{I^{\prime}, I}$;

(c) $\left(\bar{\partial}_{I}\right)_{*}: T \mathbb{V}_{I} \rightarrow \mathbb{E}_{I}$ descends to an isomorphism

$$
T \mathbb{V}_{I} /\left(\phi_{I^{\prime}, I}^{b}\right)_{*}\left(T \mathbb{V}_{I^{\prime}, I}\right) \stackrel{\sim}{\longrightarrow} \mathbb{E}_{I} / \mathbb{E}_{I^{\prime}} .
$$

(3) The composition of morphisms is defined so that $\phi_{I^{\prime \prime}, I}=\phi_{I^{\prime \prime}, I^{\prime}} \circ \phi_{I^{\prime}, I^{\prime}}$.

The following are strata compatibility conditions:

(4) (Neck length functions) For each $(i)<\left(i_{1}^{\prime}, i_{2}^{\prime}\right)$, there exists a smooth (modified) neck length function

$$
\mathfrak{n l}_{\left(i_{1}^{\prime}, i_{2}^{\prime}\right)}: \cup_{c\left(I^{\prime \prime}\right)=(i)} \widetilde{\mathcal{G}}_{\sigma}\left(\mathbb{V}_{i_{1}^{\prime \prime}}, \ldots, \mathbb{V}_{i_{k}^{\prime \prime}}\right) \rightarrow \mathbb{R}^{\geq 0}
$$

such that

$\mathbb{V}_{I^{\prime}, I}:=\left\{[u] \in \mathbb{V}_{I^{\prime}} \mid \mathcal{L}-\varepsilon^{\prime \prime} \leq \mathfrak{n l}_{\left(c\left(i_{1}, \ldots, i_{j}\right), c\left(i_{j+1}, \ldots, i_{k}\right)\right)}([u]) \leq \mathcal{L}, \forall i_{j} \in \delta\left(I, I^{\prime}\right)\right\}$.

(5) For each $I=\left(I_{1}, \ldots, I_{m}\right) \sqrt{2}$ there exists a $C^{1}$-bundle map $\left(\widetilde{\mathfrak{G}}_{I}, \mathfrak{G}_{I}\right)$ :

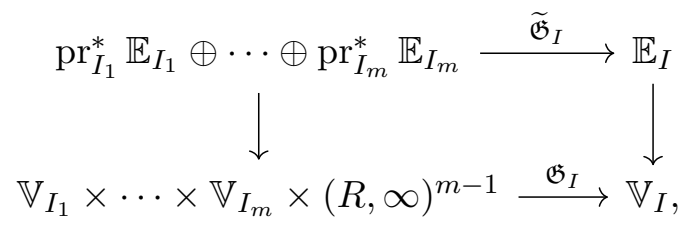

\footnotetext{
${ }^{2}$ Here we abuse notation and refer both $\left(I_{1}, \ldots, I_{m}\right)$ and $\left(i_{11}, \ldots, i_{1 j_{1}}, \ldots, i_{m 1}, \ldots, i_{m j_{m}}\right)$ by $I$, where $I_{k}=\left(i_{k 1}, \ldots, i_{k j_{k}}\right)$.
} 


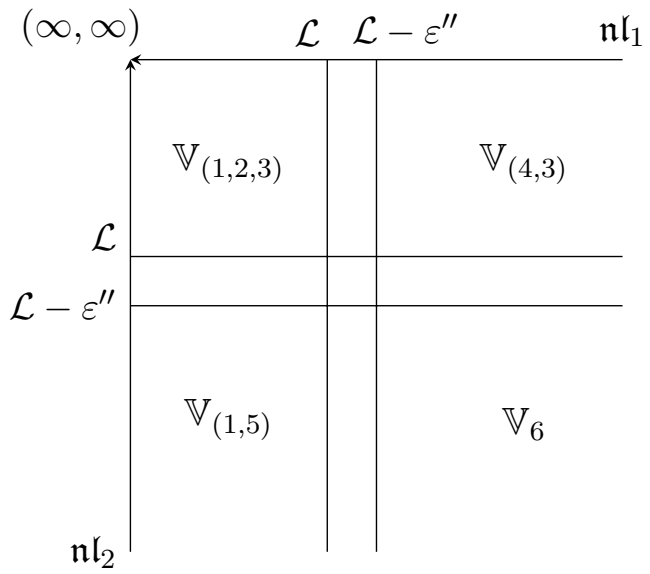

FIGURE 2. Corner structure. Suppose that $c(1,2)=4, c(2,3)=$ $5, c(4,3)=6=c(1,5)$.

where $R \gg 0, \operatorname{pr}_{I_{k}}: \mathbb{V}_{I_{1}} \times \cdots \times \mathbb{V}_{I_{m}} \times(R, \infty)^{m-1} \rightarrow \mathbb{V}_{I_{k}}$ is the projection map, $T_{j}$ is the coordinate for the $j$ th $(R, \infty)$ factor, and

(a) $\mathfrak{G}_{I}$ is a $C^{1}$-diffeomorphism onto its image;

(b) $\operatorname{Im} \mathfrak{G}_{I} \supset \mathbb{V}_{I} \cap\left\{\mathfrak{n l}_{\left(c\left(I_{1}, \ldots, I_{j}\right), c\left(I_{j+1}, \ldots, I_{m}\right)\right)} \geq R+\varepsilon^{\prime \prime}, \forall j<k\right\}$;

(c) $\mathfrak{G}_{I}\left(\left[u_{I_{1}}\right], \ldots,\left[u_{I_{m}}\right], T_{1}, \ldots, T_{m-1}\right)$ is close to the broken strip $\left(\left[u_{I_{1}}\right], \ldots,\left[u_{I_{m}}\right]\right)$;

(d) for $j=1, \ldots, m-1$, the functions $\left(\mathfrak{G}_{I}\right)_{*} T_{j}$ and $\mathfrak{n l}_{\left(c\left(I_{1}, \ldots, I_{j}\right), c\left(I_{j+1}, \ldots, I_{m}\right)\right)}$ are $C^{1}$-close;

(e) $\widetilde{\mathfrak{G}}_{I} \circ\left(\bar{\partial}_{I_{1}}, \cdots, \bar{\partial}_{I_{m}}\right)$ and $\bar{\partial}_{I} \circ \mathfrak{G}_{I}$ are $C^{1}$-close;

(f) the errors of (d) and (e) go to zero as $T_{j} \rightarrow \infty$ for all $j=1, \ldots, m-1$;

(g) $\mathfrak{G}_{\left(I_{1}, \ldots, I_{m}\right)}$ and $\mathfrak{G}_{\left(I_{1}, \ldots,\left(I_{a}, \ldots, I_{b}\right), \ldots I_{m}\right)} \circ\left(\mathrm{id}, \ldots, \mathfrak{G}_{\left(I_{a}, \ldots, I_{b}\right)}, \ldots, \mathrm{id}\right)$ are $C^{1}$-close with error $\rightarrow 0$ as $T_{j} \rightarrow \infty$ for all $j=1, \ldots, m-(b-a)-1$.

We say that $\mathscr{K}$ is $G$-invariant if, for each $g \in G, g$ induces an isomorphism

$$
\left(\mathbb{V}_{I} \rightarrow \mathbb{E}_{I}\right) \stackrel{\sim}{\longrightarrow}\left(\mathbb{V}_{g(I)} \rightarrow \mathbb{E}_{g(I)}\right)
$$

such that $\bar{\partial}_{I}, \psi_{I}, \mathfrak{n l}_{\left(i_{1}^{\prime}, i_{2}^{\prime}\right)}, \mathfrak{G}_{I}$ are taken to $\bar{\partial}_{g(I)}, \psi_{g(I)}, \mathfrak{n l}_{g\left(i_{1}^{\prime}, i_{2}^{\prime}\right)}, \mathfrak{G}_{g(I)}$.

A section of $\mathscr{K}$ is a collection $\left\{\mathfrak{s}_{I}: \mathbb{V}_{I} \rightarrow \mathbb{E}_{I}\right\}_{I}$ of obstruction sections such that:

(1) $\phi_{I^{\prime}, I}^{\sharp} \circ \mathfrak{s}_{I^{\prime}, I}=\mathfrak{s}_{I} \circ \phi_{I^{\prime}, I}^{b}$, where $\mathfrak{s}_{I^{\prime}, I}:=\left.\mathfrak{s}_{I}\right|_{\mathbb{V}_{I^{\prime}, I}} ;$

(2) $\bar{\partial}_{I}^{-1}\left(\mathfrak{s}_{I}\right) \cap \phi_{I^{\prime}, I}^{b}\left(\mathbb{V}_{I^{\prime}, I}\right)=\phi_{I^{\prime}, I}^{b}\left(\bar{\partial}_{I^{\prime}}^{-1}\left(\mathfrak{s}_{I^{\prime}, I}\right)\right)$;

(3) for each $I=\left(i_{1}, \cdots, i_{m}\right), \widetilde{\mathfrak{G}}_{I} \circ\left(\mathfrak{s}_{i_{1}}, \cdots, \mathfrak{s}_{i_{m}}\right)$ and $\mathfrak{s}_{I} \circ \mathfrak{G}_{I}$ are $C^{1}$-close and the error goes to 0 as $T_{j} \rightarrow \infty$ for all $i=1, \cdots, m-1$.

Remark 2.7.2. There is no reason to expect the sections $\left\{\mathfrak{s}_{I}\right\}_{I}$ to be $G$-invariant. This will be treated in Section 4.1 .

One can also view $\mathscr{K}$ as a functor from the index tuple category $\mathcal{I}$ to the "category of Kuranishi charts". 
Let $\mathscr{K}\left(\mathcal{M}_{i}\right)$ (also written as $\mathscr{K}(p, q ; A)$ if $\mathcal{M}_{i}=\mathcal{M}(p, q ; A)$ ) be the full subcategory of $\mathscr{K}$ with objects $I$ such that $c(I)=i$.

Given a section $\mathfrak{S}=\left\{\mathfrak{s}_{I}\right\}_{c(I)=(i)}$ of $\mathscr{K}\left(\mathcal{M}_{i}\right)$, we define

$$
\mathcal{Z}\left(\mathscr{K}\left(\mathcal{M}_{i}\right), \mathfrak{S}\right)=\left(\coprod_{c(I)=(i)} \bar{\partial}_{I}^{-1}\left(\mathfrak{s}_{I}\right)\right) / \sim \mathscr{K},
$$

where $\sim \mathscr{K}$ is the identification given by the morphisms.

We now come to an important point: There is no reason to expect $\mathcal{Z}\left(\mathscr{K}\left(\mathcal{M}_{i}\right), \mathfrak{S}\right)$ for an abstract semi-global Kuranishi structure to be a manifold, i.e., the Hausdorff property is not automatic. However, in our case the existence of the neck length functions implies the following analog of [BBH2, Lemma 8.8.1]:

Lemma 2.7.3. $\mathcal{Z}\left(\mathscr{K}\left(\mathcal{M}_{i}\right), \mathfrak{S}\right)$ is a manifold.

Proof. Same as that of [BH2, Lemma 8.8.1].

Taking limits. So far we have constructed a semi-global Kuranishi structure $\mathcal{K}$ and a section $\mathfrak{S}$ for the $G$-invariant finite set $\left\{\mathcal{M}_{1}, \ldots, \mathcal{M}_{N}\right\}$. If $\left\{\mathcal{M}_{1}, \mathcal{M}_{2}, \ldots\right\}$ is infinite, we choose increasing sequences $N_{1}, N_{2}, \cdots \rightarrow \infty$ and $\ell_{1}, \ell_{2}, \cdots \rightarrow \infty$ of integers such that $\left\{\mathcal{M}_{1}, \ldots, \mathcal{M}_{N_{j}}\right\}$ is $G$-invariant for each $N_{j}$ and construct $\mathcal{K}^{(j)}$ such that the fibers of the obstruction bundles $\mathbb{E}_{i}^{(j)} \rightarrow \mathbb{V}_{i}^{(j)}$ (i.e., $\mathbb{E}_{i} \rightarrow \mathbb{V}_{i}$ for $j$ ) are $e^{q, \ell_{j}}$, where $q=\mathbf{t}\left(\mathcal{M}_{i}\right)$. Since $e^{q, \ell_{j}}$ naturally includes into $e^{q, \ell_{j+1}}$, there are natural inclusions

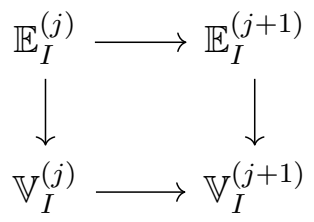

that commute with the morphisms $\phi_{I^{\prime}, I}^{(j)}$ and $\phi_{I^{\prime}, I}^{(j+1)}$. Assuming we have already constructed the section $\mathfrak{S}^{(j)}$, we construct $\mathfrak{S}^{(j+1)}$ such that $\mathfrak{s}_{I}^{(j+1)}$ is the image of $\mathfrak{s}_{I}^{(j)}$ under the appropriate inclusions $\oplus_{q} e^{q, \ell_{j}} \rightarrow \oplus_{q} e^{q, \ell_{j+1}}$ whenever the entries of $I$ are $\leq N_{j}$. This is sufficient to ensure that, for $i \leq N_{j}$, there is a natural identification

$$
\mathcal{Z}\left(\mathscr{K}^{(j)}\left(\mathcal{M}_{i}\right), \mathfrak{S}^{(j)}\right) \simeq \mathcal{Z}\left(\mathscr{K}^{(j+1)}\left(\mathcal{M}_{i}\right), \mathfrak{S}^{(j+1)}\right) .
$$

We write $\mathcal{Z}\left(\mathscr{K}\left(\mathcal{M}_{i}\right), \mathfrak{S}\right)$ for any of the $\mathcal{Z}\left(\mathscr{K}^{(j)}\left(\mathcal{M}_{i}\right), \mathfrak{S}^{(j)}\right)$ such that $i \leq N_{j}$.

From now on we will assume $\left\{\mathcal{M}_{1}, \mathcal{M}_{2}, \ldots\right\}$ is finite, making the appropriate modifications as above, if it is not.

Implicit charts. Our semi-global Kuranishi structure $\mathscr{K}\left(\mathcal{M}_{i}\right), \mathcal{M}_{i}=\mathcal{M}\left(p_{i}, q_{i} ; A_{i}\right)$, can be converted into a single global implicit chart in the sense of Pardon [Pa]. Let $\mathcal{S}\left(p_{i}, q_{i}\right)$ be the set of all the $q_{j}$ that appear before $\left(p_{i}, q_{i}, A_{i}\right)$ in the list (2.5.2) and take the global fiber to be

$$
e\left(p_{i}, q_{i}\right):=\oplus_{r \in \mathcal{S}\left(p_{i}, q_{i}\right)} e^{r, \ell}
$$


We consider solutions $(u, \xi)$,

$$
u \in \cup_{c\left(i_{1}, \ldots, i_{k}\right)=(i)} \widetilde{\mathcal{G}}_{\sigma}\left(\mathbb{V}_{i_{1}}, \ldots, \mathbb{V}_{i_{k}}\right), \quad \xi=\left(\xi_{r}\right)_{r \in \mathcal{S}\left(p_{i}, q_{i}\right)} \in e\left(p_{i}, q_{i}\right),
$$

to the equation

$$
\bar{\partial} u=\sum_{r \in \mathcal{S}\left(p_{i}, q_{i}\right)}\left(\zeta \circ \mathfrak{n l}_{r}(u)\right) \cdot \xi_{r},
$$

where $\zeta$ and $\mathfrak{n l}_{r}$ are as given in Definitions 2.5.3 and 2.5.6. Roughly speaking, we turn off the perturbations for $e^{r, \ell}$ when $\mathfrak{n l}_{r}(u) \leq \mathcal{L}$ but still remember the data for $e^{r, \ell}$.

\subsection{Equivariant semi-global Kuranishi structures for chain maps and chain homotopies.}

2.8.1. Chain maps. Let $H_{s}: M \rightarrow \mathbb{R}, s \in[0,1]$, be a compactly supported, time-dependent, $G$-invariant Hamiltonian function and let $\phi_{s}, s \in[0,1]$, be the corresponding 1-parameter family of Hamiltonian symplectomorphisms of $(M, \omega)$ with $\phi_{0}=\mathrm{id}$; we call such a $\phi_{s}$ a $G$-equivariant Hamiltonian isotopy. Writing $L_{i}^{\prime}=\phi_{1}\left(L_{i}\right), i=0,1$, we assume that $L_{0}^{\prime} \pitchfork L_{1}^{\prime}$. Let $\left\{J^{s}\right\}_{s \in[0,1]}$ be a 1-parameter family of almost complex structures that are $\omega$-compatible, $G$-invariant, and satisfy $(\mathbf{J})$.

Define a smooth function $\vartheta_{0}: \mathbb{R} \rightarrow[0,1]$ such that $\vartheta_{0}(s)=0$ for $s \leq 0$ and $\vartheta_{0}(s)=1$ for $s \geq 1$.

Given $p \in L_{0} \cap L_{1}, q \in L_{0}^{\prime} \cap L_{1}^{\prime}$, and $A \in \pi_{2}(p, q)$, let $\mathcal{M}^{\circ}(p, q ; A)$ (we are suppressing $\left.\left\{J^{s}\right\}\right)$ be the space of smooth maps $u: \mathbb{R} \times[0,1] \rightarrow M$ that satisfy (A3) and (A4), in addition to:

$\left(\mathrm{A}^{\prime}\right) u_{s}(s, t)+J^{\vartheta_{0}(s)}(u(s, t)) u_{t}(s, t)=0$, and

$\left(\mathrm{A} 2^{\prime}\right) u(s, 0) \in \phi_{\vartheta_{0}(s)}\left(L_{0}\right)$ and $u(s, 1) \in \phi_{\vartheta_{0}(s)}\left(L_{1}\right)$.

When we are defining chain maps and chain homotopies, the moduli spaces for $\left(L_{0}, L_{1}\right)$ will have superscripts - as in $\mathcal{M}^{-}\left(p, p^{\prime} ; A\right)$ and the moduli spaces for $\left(L_{0}^{\prime}, L_{1}^{\prime}\right)$ will have superscripts + as in $\mathcal{M}^{+}\left(q, q^{\prime} ; A\right)$.

The construction of the Kuranishi charts and the Kuranishi structure from Sections 2.4 to 2.7 carry over with very few modifications: Under our assumptions there are finitely many moduli spaces of type $\mathcal{M}^{\circ}(p, q ; A), \mathcal{M}^{-}(p, q ; A)$, and $\mathcal{M}^{+}(p, q ; A)$, which we list as

$$
\mathcal{M}_{1}, \ldots, \mathcal{M}_{\rho}
$$

as before so that $\omega(A)$ is in nondecreasing order. The type of $\mathcal{M}_{i}$ is given by the superscript $\circ,-$, or + .

Definition 2.8.1. A tuple $I=\left(i_{1}, \ldots, i_{k}\right)$ is a $c$-index tuple (where $c$ stands for chain map), if it satisfies the conditions of Definition 2.5.4 and

- there exists $i_{j}$ such that $\mathcal{M}_{i_{j}}$ has type $\circ$ and all $i_{l}$ with $l<j$ have type and all $i_{l}$ with $l>j$ have type + . 
The charts $\left(\pi_{I}: \mathbb{E}_{I} \rightarrow \mathbb{V}_{I}, \bar{\partial}_{I}, \psi_{I}\right)$ are constructed in exactly the same way as before, where $I$ is now a $c$-index tuple. By construction the Kuranishi structure is $G$-invariant.

2.8.2. Chain homotopies. Fix $T \gg 0$. We define a smooth function

$$
\Theta: \mathbb{R} \times[0,1] \rightarrow[0,1]
$$

with coordinates $(s, \tau)$ for $\mathbb{R} \times[0,1]$ such that:

- $\Theta(s, 0)=1$ for $s \in[-T+1, T-1]$;

- $\Theta(s, 1)=0$ for all $s$;

- $\Theta(s, \tau)=0$ for all $s>T$ and $s<-T$ and $\tau \in[0,1]$.

For each $\tau \in[0,1]$, let $\mathcal{M}_{\tau}^{\circ}(p, q ; A)$ be the space of smooth maps $u: \mathbb{R} \times$ $[0,1] \rightarrow M$ that satisfy (A3), (A4),

$\left(\mathrm{A}^{\tau}\right) u_{s}(s, t)+J^{\Theta(s, \tau)}(u(s, t)) u_{t}(s, t)=0$, and

$\left(\mathrm{A} 2^{\tau}\right) u(s, 0) \in \phi_{\Theta(s, \tau)}\left(L_{0}\right)$ and $u(s, 1) \in \phi_{\Theta(s, \tau)}\left(L_{1}\right)$.

We also write

$$
\mathcal{M}_{\{\tau\}}^{\circ}(p, q ; A)=\coprod_{\tau \in[0,1]} \mathcal{M}_{\tau}^{\circ}(p, q ; A) .
$$

For each $c$-index tuple and each $\tau \in[0,1]$ we construct a chart

$$
\left(\pi_{I, \tau}: \mathbb{E}_{I, \tau} \rightarrow \mathbb{V}_{I, \tau}, \bar{\partial}_{I, \tau}, \psi_{I, \tau}\right),
$$

which can be combined into a family

$$
\left(\pi_{I,[0,1]}: \mathbb{E}_{I,[0,1]} \rightarrow \mathbb{V}_{I,[0,1]}, \bar{\partial}_{I,[0,1]}, \psi_{I,[0,1]}\right)
$$

By construction the family of Kuranishi structures is $G$-invariant.

\section{ORIENTATIONS}

The goal of this section is to review the definition of a coherent (= compatible with gluing) system of orientations on the moduli space of (finite energy) $J$ holomorphic strips for a pair $\left(L_{0}, L_{1}\right)$ of Lagrangians, following [FO3] and then adapt it to the case with a $G$-action. We will see that in general, $g \in G$ only preserves the orientation of $\mathcal{M}(p, q ; A)$ up to a sign $\sigma(g, p, q) \in\{-1,1\}$ that is independent of $A$. But this is enough to define a $G$-action on the $C F^{\bullet}\left(L_{0}, L_{1}\right)$.

3.1. Cauchy-Riemann tuples. A Cauchy-Riemann tuple is a quadruple $(\Sigma, \xi, \eta, D)$ satisfying (CR1)-(CR4):

(CR1) $\Sigma=B \backslash X$, where $B$ is the closed unit disk in $\mathbb{C}$ and $X$ is a finite subset of $\partial B$.

For each $x \in X$, let $I_{x} \subset \partial B$ be a small interval neighborhood of $x$ and let $I_{x-}$ and $I_{x+}$ be the two connected components of $I_{x} \backslash x$.

(CR2) $\xi$ is a trivial $\mathbb{C}$-vector bundle over $\bar{\Sigma}=B$.

(CR3) $\eta$ is a real subbundle of $\left.\xi\right|_{\partial \Sigma-X}$ such that $\left.\eta\right|_{I_{x \pm}}$ extends smoothly to a real subspace $\eta_{x \pm} \subset \xi_{x}$ over $x$. Moreover, $\xi_{x}=\eta_{x+} \oplus \eta_{x-}$. 
Let $\Gamma(\Sigma, \xi)$ be the space of compactly supported smooth sections of $\left.\xi\right|_{\Sigma}$ that restrict to sections of $\eta$ along $\partial \Sigma \backslash X$. For each $x \in X$, choose a neighborhood $N(x) \subset B$ and a holomorphic identification of $\Sigma \cap N(x)$ with a strip-like end $[0, \infty) \times[0,1]$ with coordinates $(s, t)$. Let $W^{k+1, p}(\Sigma, \xi)$ be the closure of $\Gamma(\Sigma, \xi)$ in the $W^{k+1, p_{-}}$ norm with respect to a metric on $\Sigma$ consistent with the strip-like ends and a metric on $\xi$. The space $W^{k, p}\left(\Sigma, \wedge^{0,1} \Sigma \otimes_{\mathbb{C}} \xi\right)$ is defined similarly.

(CR4) The operator $D: W^{k+1, p}(\Sigma, \xi) \rightarrow W^{k, p}\left(\Sigma, \wedge^{0,1} \Sigma \otimes_{\mathbb{C}} \xi\right)$ is a real-linear Cauchy-Riemann operator such that on each strip-like end

$$
D w=\frac{1}{2}\left(\nabla_{s} w+J \nabla_{t} w\right) \otimes(d s+i d t),
$$

where $J$ is the complex structure on $\xi$ and $\nabla$ is a connection of $\xi$.

See [MS2, Appendix C] for the definition of a real-linear Cauchy-Riemann operator over a compact Riemann surface.

3.2. Auxiliary orientation data. Recall the determinant line of $(\Sigma, \xi, \eta, D)$ is a 1-dimensional vector space defined by

$$
\operatorname{det} D:=\wedge^{\text {top }} \operatorname{ker} D \otimes_{\mathbb{R}} \wedge^{\text {top }}(\operatorname{coker} D)^{*} \text {. }
$$

Let $\pi: \mathbb{E}(p, q ; A) \rightarrow \mathbb{V}(p, q ; A)$ be an interior semi-global Kuranishi chart for $\mathcal{M}(p, q ; A)$. Given $u$ with $[u] \in \mathbb{V}$, we define the Cauchy-Riemann tuple

$$
\left(\Sigma^{u}, \xi^{u}, \eta^{u}, D^{u}\right):=\left(S, u^{*} T M, \sqcup_{i \in\{0,1\}} u(\cdot, i)^{*} T L_{i}, D_{u}\right),
$$

where $S=\mathbb{R} \times[0,1]$ and $D^{u}$ is the linearized $\bar{\partial}$-operator at $u$.

A coherent system of orientations $\mathfrak{o}\left(D^{u}\right)$ of $\operatorname{det} D^{u}$ will depend on the following auxiliary orientation data; see Theorem 3.4.1.

Definition 3.2.1. A choice of auxiliary orientation data consists of:

(O1) a relative spin structure for the pair $\left(L_{0}, L_{1}\right)$; and for each $p \in L_{0} \cap L_{1}$,

(O2) a capping Lagrangian path;

(O3) a capping orientation; and

(O4) a stable capping trivialization.

We will explain $(\mathrm{O} 2)$ and $(\mathrm{O} 3)$, leaving $(\mathrm{O} 1)$ and $(\mathrm{O} 4)$ for the next subsection.

A capping Lagrangian path $(\mathrm{O} 2)$ is a path $\left\{\mathcal{L}_{p, t}\right\}_{0 \leq t \leq 1}$ in the oriented Lagrangian Grassmannian $\operatorname{Lag}\left(T_{p} M, \omega_{p}\right)$ such that $\mathcal{L}_{p, i}=\bar{T}_{p} L_{i}$ with orientations, for $i=0,1$.

For each $p \in L_{0} \cap L_{1}$, we define a Cauchy-Riemann tuple $\left(\Sigma^{p+}, \xi^{p+}, \eta^{p+}, D^{p+}\right)$ as follows: Let $\Sigma^{p+}$ be the closed unit disk in $\mathbb{C}$ with one boundary puncture, identified with the upper half plane $\mathbb{H}=\{z \mid \operatorname{Im} z \geq 0\}$, and let $\pi_{p}: \Sigma^{p+} \rightarrow M$ be the constant map to $p$. We then define:

- $\xi^{p+}=\pi_{p}^{*}\left(T_{p} M\right)$,

- $\eta_{z}^{p+}=\mathcal{L}_{p, 0}$ for $z \in(-\infty, 0), \eta_{z}^{p+}=\mathcal{L}_{p, z}$ for $z \in[0,1]$, and $\eta_{z}^{p+}=\mathcal{L}_{p, 1}$ for $z \in(1,+\infty)$, and

- $D^{p+}$ is a fixed real linear Cauchy-Riemann operator (the choice is unique up to homotopy). 
We can similarly choose the Cauchy-Riemann tuple $\left(\Sigma^{p-}, \xi^{p-}, \eta^{p-}, D^{p-}\right)$ by swapping the roles of $L_{0}$ and $L_{1}$.

Finally, a capping orientation (O3) is a choice of orientation $\mathfrak{o}\left(D^{p+}\right)$ (but not $\left.\mathfrak{o}\left(D^{p-}\right)\right)$.

3.3. Relative spin structures. A pair $\left(L_{0}, L_{1}\right)$ is relatively spin if there exists $s t \in H^{2}(M ; \mathbb{Z} / 2)$ such that $w_{2}\left(T L_{i}\right)=\iota_{i}^{*} s t$ for $i=0,1$ : Fix a triangulation $\tau$ of $M$ such that $L_{0}, L_{1}$, and $L_{0} \cap L_{1}$ are subcomplexes. Choose an oriented real vector bundle $V$ of rank $\geq 2$ on the 3 -skeleton $M^{(3)}$ of $M$ such that $w_{2}(V)=$ st. (Here we are using the notation $X^{(i)}$ for the $i$-skeleton of a triangulation of $X$.) Then the bundle $\left.\left.T L_{i}\right|_{L_{i}^{(2)}} \oplus V\right|_{L_{i}^{(2)}}$ is spin and hence is a trivial bundle. Choosing a spin structure is equivalent to choosing a homotopy class of trivializations $\boldsymbol{t}_{i}$ of $\left.\left.T L_{i}\right|_{L_{i}^{(1)}} \oplus V\right|_{L_{i}^{(1)}}$ that extends to $L_{i}^{(2)}$. Since $\pi_{2}(S O(m))=0$ for $m \geq 3$, the extension to $L_{i}^{(2)}$ is unique and $\mathfrak{t}_{i}$ also extends to $L_{i}^{(3)}$. We will refer to choices of $\tau, V$, and homotopy classes of $\mathfrak{t}_{i}, i=0,1$, as a relative spin structure; see [FO3, Section 8.1] for an explanation of when two relative spin structures are equivalent. A more algebraic (and cleaner) definition of a relative spin structure can be found in [WW, Section 3.1] and [SC].

Let $\widetilde{\mathcal{L}}_{p} \rightarrow[0,1]$ be a vector bundle whose fiber over $t \in[0,1]$ is $\mathcal{L}_{p, t} \oplus V_{p}$. Then a stable capping trivialization $(\mathrm{O} 4)$ is a trivialization $\widetilde{\mathfrak{t}}_{p}$ of $\widetilde{\mathcal{L}}_{p}$ that agrees with the trivializations $\mathfrak{t}_{i}$ of $\left.\left(\left.\left.T L_{i}\right|_{L_{i}^{(1)}} \oplus V\right|_{L_{i}^{(1)}}\right)\right|_{p}$ that we have already chosen for $i=0,1$.

3.4. Coherent orientation system. We review the following theorem from [FO3, Section 8.1]:

Theorem 3.4.1. The moduli space of J-holomorphic strips admits a coherent orientation system if the pair of Lagrangians $\left(L_{0}, L_{1}\right)$ is relative spin. Moreover, the choice of auxiliary orientation data $(\mathrm{Ol})(\mathrm{O} 4)$ determines the orientation.

We give a sketch of the proof, partly to establish notation. The fundamental fact that we use is the following (cf. [FO3, Proposition 34.3]), stated without proof.

Fact 3.4.2. Given a Cauchy-Riemann tuple $\left(\Sigma, \mathbb{C}^{n}, \eta, D\right)$, if $\Sigma$ has no punctures and $\eta$ is trivial, then any trivialization of $\eta$ canonically determines an orientation of $\operatorname{det} D$.

\section{Sketch of proof of Theorem 3.4.1}

Step 1. Let $\left(\Sigma^{1}, \xi^{1}, \eta^{1}, D^{1}\right)$ and $\left(\Sigma^{2}, \xi^{2}, \eta^{2}, D^{2}\right)$ be two Cauchy-Riemann tuples. Given punctures $x_{1} \in \partial \Sigma^{1}$ and $x_{2} \in \partial \Sigma^{2}$, suppose there is a $\mathbb{C}$-linear isomorphism $\Phi: \xi_{x_{1}}^{1} \stackrel{\sim}{\longrightarrow} \xi_{x_{2}}^{2}$ that maps $\eta_{x_{1} \pm}^{1}$ to $\eta_{x_{2} \mp}^{2}$. Then there is an associated Cauchy-Riemann tuple $\left(\Sigma^{1,2}, \xi^{1,2}, \eta^{1,2}, D^{1,2}\right)$ defined by a straightforward pregluing which identifies $x_{1}$ and $x_{2}$ and the orientations of $\operatorname{det} D^{1}$ and $\operatorname{det} D^{2}$ induce an orientation of $\operatorname{det} D^{1,2}$.

In particular, if we preglue $\left(\Sigma^{q+}, \xi^{q+}, \eta^{q+}, D^{q+}\right)$ and $\left(\Sigma^{q-}, \xi^{q-}, \eta^{q-}, D^{q-}\right)$, we obtain the Cauchy-Riemann tuple $\left(\Sigma^{q+, q-}, \xi^{q+, q-}, \eta^{q+, q-}, D^{q+, q-}\right)$ and it has a canonical orientation by Fact 3.4.2. (Here we are taking the trivializations of $\eta^{q+}$ 
and $\eta^{q-}$ to come from the same trivialization of $\mathcal{L}_{p}$; then the trivialization of $\eta^{q+, q-}$ is independent of the choice of trivialization of $\mathcal{L}_{p}$.) Hence the capping orientation $\mathfrak{o}\left(D^{q+}\right)$ determines $\mathfrak{o}\left(D^{q-}\right)$.

For any $u$ with $[u] \in \mathbb{V}(p, q, A)$, we preglue

$$
\left(\Sigma^{p+}, \xi^{p+}, \eta^{p+}, D^{p+}\right), \quad\left(\Sigma^{u}, \xi^{u}, \eta^{u}, D^{u}\right), \quad\left(\Sigma^{q-}, \xi^{q-}, \eta^{q-}, D^{q-}\right)
$$

along $p$ and $q$ to obtain

$$
\left(\Sigma^{p+, u, q-}, \xi^{p+, u, q-}, \eta^{p+, u, q-}, D^{p+, u, q-}\right) .
$$

If we can orient $\operatorname{det}\left(D^{p+, u, q-}\right)$, then $\mathfrak{o}\left(D^{u}\right)$ is determined by $\mathfrak{o}\left(D^{p+, u, q-}\right)$ and the capping orientations $\mathfrak{o}\left(D^{p+}\right)$ and $\mathfrak{o}\left(D^{q-}\right)$.

Step 2. By the simplicial approximation theorem, after a homotopy we can assume that $u(\Sigma) \subseteq M^{(2)}$ and $u(\partial \Sigma) \subseteq L_{0}^{(1)} \cup L_{1}^{(1)}$. Let $V \rightarrow M^{(3)}$ be the bundle appearing in the definitions of $(\mathrm{O} 1)$ and $(\mathrm{O} 4)$.

Define Cauchy-Riemann tuples

$$
\left(\Sigma^{p+}, \xi_{V}^{p+}, \eta_{V}^{p+}, D_{V}^{p+}\right), \quad\left(\Sigma^{u}, \xi_{V}^{u}, \eta_{V}^{u}, D_{V}^{u}\right), \quad\left(\Sigma^{p-}, \xi_{V}^{p-}, \eta_{V}^{p-}, D_{V}^{p-}\right)
$$

in the same way as the versions without $V$, except that we replace $T M$ by $V \oplus i V$, $T L_{i}$ by $V$ for $i=0,1$, and $\mathcal{L}_{p, t}$ by $V_{p}$. By pregluing as in Step 1, we obtain

$$
\left(\Sigma^{p+, u, q-}, \xi_{V}^{p+, u, q-}, \eta_{V}^{p+, u, q-}, D_{V}^{p+, u, q-}\right) .
$$

A key point to observe now is that, since $V$ is oriented and defined over $u(\Sigma)$, there is a canonical equivalence class of trivializations of $\eta_{V}^{p+, u, q-}$ and hence a canonical orientation of $\operatorname{det} D_{V}^{p+, u, q-}$ by Fact 3.4 .2

We take the direct sum of

$$
\left(\xi^{p+, u, q-}, \eta^{p+, u, q-}, D^{p+, u, q-}\right) \quad \text { and } \quad\left(\xi_{V}^{p+, u, q-}, \eta_{V}^{p+, u, q-}, D_{V}^{p+, u, q-}\right),
$$

over $\Sigma^{p+, u, q-}$ to obtain

$$
\left(\Sigma^{p+, u, q-}, \xi^{p+, u, q-} \oplus \xi_{V}^{p+, u, q-}, \eta^{p+, u, q-} \oplus \eta_{V}^{p+, u, q-}, D^{p+, u, q-} \oplus D_{V}^{p+, u, q-}\right) .
$$

Now $\mathfrak{t}_{i}, \widetilde{\mathfrak{t}}_{p}$, and $\widetilde{\mathfrak{t}}_{q}$ give a trivialization of $\eta^{p+, u, q-} \oplus \eta_{V}^{p, u, q-}$, so $\operatorname{det}\left(D^{p+, u, q-} \oplus\right.$ $\left.D_{V}^{p+, u, q-}\right)$ is canonically oriented by Fact 3.4.2 $\operatorname{Since} \operatorname{det}\left(D^{p+, u, q-} \oplus D_{V}^{p+, u, q-}\right)$ is canonically isomorphic to $\operatorname{det} D^{p+, u, q-} \otimes \operatorname{det} D_{V}^{p+, u, q-}$ and $\operatorname{det} D_{V}^{p+, u, q-}$ is canonically orientated, we obtain a canonical orientation of $\operatorname{det} D^{p+, u, q-}$.

Step 3. It remains to show that $\mathfrak{o}\left(D^{u}\right)$ is independent of the choices. We refer the reader to [FO3, Section 8.1] for a proof.

Since $\operatorname{det} D^{u}$ is canonically isomorphic to $\operatorname{det} \mathbb{D}^{u}$, where $\mathbb{D}^{u}$ is the linearized operator of $\bar{\partial}_{J}: \mathbb{V}(p, q ; A) \rightarrow \mathbb{E}(p, q ; A)$, a choice of auxiliary orientation data induces a system of orientations on

$$
\left(\Lambda^{\mathrm{top}} \mathbb{E}_{I}\right)^{*} \otimes \Lambda^{\mathrm{top}} T \mathbb{V}_{I}
$$

Next we study orientations under the group action. To do that, we first need to allow $G$ to act on the obstruction bundle. 


\subsection{Orientations on $e^{r, \ell}$.}

Lemma 3.5.1. If $\ell$ is an even multiple of $n$, then $e^{r, \ell}$ admits a canonical $G$ invariant orientation.

Proof. Without loss of generality, we assume:

(i) $T_{r} M \simeq \mathbb{R}^{n} \oplus i \mathbb{R}^{n}=\mathbb{C}^{n}$, where $T_{r} L_{0}$ is the $\mathbb{R}^{n}$ factor and $T_{r} L_{1}$ is the $i \mathbb{R}^{n}$ factor;

(ii) $J(r)=J_{0}$ is the standard complex structure that takes $v \in \mathbb{R}^{n}$ to $i v \in i \mathbb{R}^{n}$ and $g_{r}$ is the standard Euclidean structure on $T_{r} M$; and

(iii) $G$ leaves $T_{r} L_{0}$ invariant. Since $G$ is compatible with $J$ and $g$, it can be described by a representation $\rho: G \rightarrow O\left(\mathbb{R}^{n}\right)$.

The asymptotic operator $A$ is given by $-J_{0} \frac{\partial}{\partial t}$ with boundary conditions $\mathbb{R}^{n}$ at $t=0$ and $i \mathbb{R}^{n}$ at $t=1$. For each $k=0,1, \ldots$, there are $n$ eigenfunctions

$$
\tilde{e}_{j}^{k}:[0,1] \rightarrow \mathbb{C}^{n}, \quad t \mapsto e^{i(\pi k+\pi / 2) t} e_{j}, \quad j=1, \ldots, n,
$$

where $e_{1}, \ldots, e_{n}$ is a basis for $\mathbb{R}^{n}$. Writing $\ell=2 k_{0} n$, we choose the orientation

$$
\tilde{e}_{1}^{0} \wedge \cdots \wedge \tilde{e}_{n}^{0} \wedge \cdots \wedge \tilde{e}_{1}^{2 k_{0}-1} \wedge \cdots \wedge \tilde{e}_{n}^{2 k_{0}-1}
$$

for $e^{r, \ell}$. Since $G$ acts on each $\mathbb{R}\left\langle\tilde{e}_{1}^{k}, \ldots, \tilde{e}_{n}^{k}\right\rangle$ in the same way as on $\mathbb{R}^{n}$ using the identification $\tilde{e}_{j}^{k} \mapsto e_{j}$, for any $g \in G$,

$g\left(\tilde{e}_{1}^{0}\right) \wedge \cdots \wedge g\left(\tilde{e}_{n}^{0}\right) \wedge \cdots \wedge g\left(\tilde{e}_{1}^{2 k_{0}-1}\right) \wedge \cdots \wedge g\left(\tilde{e}_{n}^{2 k_{0}-1}\right)=\tilde{e}_{1}^{0} \wedge \cdots \wedge \tilde{e}_{n}^{0} \wedge \cdots \wedge \tilde{e}_{1}^{2 k_{0}-1} \wedge \cdots \wedge \tilde{e}_{n}^{2 k_{0}-1}$

and $G$ preserves the orientation. Note that the definition in Equation 3.5.1 does not depend on the orientation of $\mathbb{R}^{n}$.

From now on let us assume that $\ell$ is an even multiple of $n$ and hence all the $e^{r, \ell}$ are canonically oriented, so $G$ acts on $\mathbb{E}_{I} \rightarrow \mathbb{V}_{I}$.

3.6. Orientations under group action. Now we study the action of $G$ on the orientation of $\left(\Lambda^{\text {top }} \mathbb{E}_{I}\right)^{*} \otimes \Lambda^{\text {top }} T \mathbb{V}_{I}$.

We assume Condition $(\mathbf{O})$ from Section 17 i.e., that the relative spin structure is preserved under $G$, whose definition we give presently.

Let $\left(\tau, V, \mathfrak{t}_{0}, \mathfrak{t}_{1}\right)$ be a relative spin structure for $\left(L_{0}, L_{1}\right)$. Let $\tau$ be a $G$-equivariant triangulation of $M$; such a triangulation exists by the equivariant triangulation theorem. Then $\left(\tau, V, \mathfrak{t}_{0}, \mathfrak{t}_{1}\right)$ is preserved by $G$, if for any $g \in G$, there exists an orientation-preserving bundle isomorphism $\theta_{g}: V \stackrel{\sim}{\longrightarrow} V$ such that

- $\pi_{V} \circ \theta_{g}=g \circ \pi_{V}$, where $\pi_{V}: V \rightarrow M^{(3)}$ is the projection to the base, and

- for each $i=0,1$, the trivialization

$$
\mathfrak{t}_{i}:\left.\left.T L_{i}\right|_{L_{i}^{(2)}} \oplus V\right|_{L_{i}^{(2)}} \rightarrow L_{i}^{(2)} \times \mathbb{R}^{d}
$$

is homotopic to

$$
g_{\sharp} \mathfrak{t}_{i}:=\mathfrak{t}_{i} \circ\left(\left.g_{*} \oplus \theta_{g}\right|_{L_{i}^{(2)}}\right)^{-1},
$$

where $d=n+\operatorname{rank} V$ and

$$
\left.g_{*} \oplus \theta_{g}\right|_{L_{i}^{(2)}}:\left.\left.\left.\left.T L_{i}\right|_{L_{i}^{(2)}} \oplus V\right|_{L_{i}^{(2)}} \rightarrow T L_{i}\right|_{L_{i}^{(2)}} \oplus V\right|_{L_{i}^{(2)}} .
$$


For $p \in L_{0} \cap L_{1}$ and $g \in G$, let $s=g p$. At $s$, we have the canonical isomorphism

$$
\operatorname{det} D^{s+} \otimes \operatorname{det} D^{s-} \otimes \operatorname{det} D_{V}^{s+, s-} \simeq \operatorname{det}\left(D^{s+, s-} \oplus D_{V}^{s+, s-}\right)
$$

coming from gluing. Let $\mathfrak{o}\left(D^{p+}\right)$ and $\mathfrak{o}\left(D^{s-}\right)$ be the capping orientations of $\operatorname{det} D^{p+}$ and $\operatorname{det} D^{s-}$ and let $\mathfrak{o}\left(D_{V}^{s+, s-}\right)$ be the canonical orientation of $D_{V}^{s+, s-}$. Then $g_{\#} \mathfrak{o}\left(D^{p+}\right) \otimes \mathfrak{o}\left(D^{s-}\right) \otimes \mathfrak{o}\left(D_{V}^{s+, s-}\right)$ determines an orientation of the left-hand side of Equation (3.6.1). On the right-hand side of Equation (3.6.1), we have a canonical orientation of $\operatorname{det}\left(D^{s+, s-} \oplus D_{V}^{s+, s-}\right)$ coming from the concatenation of the stable capping trivializations $g_{\#} \widetilde{\mathfrak{t}}_{p}$ and $\widetilde{\mathfrak{t}}_{s}$. (The trivializations $g_{\#} \widetilde{\mathfrak{t}}_{p}$ and $\widetilde{\mathfrak{t}}_{s}$ a priori do not agree at the endpoints. We assume that $g_{\#} \mathfrak{t}_{i}$ has been homotoped to $\mathfrak{t}_{i}$ and by abuse of notation we refer to $g_{\#} \widetilde{\mathfrak{t}}_{p}$ as the result of applying the homotopy to $g_{\#} \widetilde{\mathfrak{t}}_{p}$.) We compare these two orientations via the isomorphism of Equation (3.6.1), and define $\sigma(p, g) \in\{ \pm 1\}$ to be the difference. For $u$ with $[u] \in \mathbb{V}(p, q, A)$, let $\mathfrak{o}\left(D^{u}\right)$ be the orientation of $u$ determined by the auxiliary orientation data (O1)$(\mathrm{O} 4)$, Then one can check that $g_{\#} \mathfrak{o}\left(D^{u}\right)=\sigma(p, g) \sigma(q, g) \mathfrak{o}\left(D^{g u}\right)$.

In general, $g \in G$ may not preserve the orientation, but we can define the action of $g \in G$ on $C F^{\bullet}\left(L_{0}, L_{1}\right)$ by sending $[p]$ to $\sigma(p, g)[g p]$. In the case when the moduli spaces that we count to define the differential $d$ of $C F^{\bullet}\left(L_{0}, L_{1}\right)$ are $G$ invariant, it is obvious that the $G$-action on $C F^{\bullet}\left(L_{0}, L_{1}\right)$ commutes with $d$. In Section 4, we see this is true even when the moduli space is not $G$-invariant.

From now on, we fix a choice of auxiliary orientation data (O1)(O4) such that the relative spin structure $(O 1)$ is preserved under the $G$-action. This gives an orientation of $\left(\Lambda^{\text {top }} \mathbb{E}_{I}\right)^{*} \otimes \Lambda^{\text {top }} T \mathbb{V}_{I}$. Since the fiber of $\mathbb{E}_{I} \rightarrow \mathbb{V}_{I}$ is canonically oriented by Section 3.5, we also get an orientation of $\mathbb{V}_{I}$.

\section{EQuivariance of CURVE COUNTING}

\subsection{Equivariance of curve counting.}

Choice of $\mathfrak{S}$. We first describe how to choose $\mathfrak{S}=\left\{\mathfrak{s}_{I}\right\}_{I}$ to be as $G$-equivariant as possible. First decompose $L_{0} \cap L_{1}$ into a disjoint union of $G$-orbits $\mathcal{O}_{p}, p \in L_{0} \cap L_{1}$. Given $\mathcal{O}_{p}$, pick a generic $s_{p} \in e^{p, \ell}$ which is sufficiently close to the origin and for each $q \in \mathcal{O}_{p}$ choose a single $g \in G$ such that $g(p)=q$ and set $s_{q}=g\left(s_{p}\right) \in e^{q, \ell}$. We then choose $\mathfrak{s}_{i}=s_{p} \in e^{p, \ell}$, where $p=\mathbf{t}\left(\mathcal{M}_{i}\right)$, and construct $\mathfrak{s}_{I}$ as described in Sections 2.4 and 2.5. We additionally assume that:

(*) $\left|s_{i}\right| \ll\left|s_{j}\right|$ if $i>j$.

Remark 4.1.1. Note that $\mathfrak{S}$ is not expected to equal $g(\mathfrak{S})$ for all $g \in G$. If we replace $\mathfrak{S}$ by a $G$-equivariant collection of multisections, the Floer chain groups will be defined over $\mathbb{Q}$ as in Cho-Hong $[\overline{\mathrm{CH}}]$. Since this leads to some loss of information, we choose to work with collections of sections.

The following key theorem makes the equivariant count work.

Theorem 4.1.2. If $\operatorname{vdim} \mathcal{M}_{i}=0$ and $g\left(\mathcal{M}_{i}\right)=\mathcal{M}_{i}$, then $\mathcal{Z}\left(\mathscr{K}\left(\mathcal{M}_{i}\right)\right.$, S) and $\mathcal{Z}\left(\mathscr{K}\left(\mathcal{M}_{i}\right), g(\mathfrak{S})\right)$ are cobordant. 
Proof. If $\overline{\mathcal{M}}_{i}=\mathcal{M}_{i}$, i.e., there is no boundary, then $\mathcal{Z}\left(\mathscr{K}\left(\mathcal{M}_{i}\right), \mathfrak{S}\right)$ is given by the preimage of $s_{r}, r=\mathbf{t}\left(\mathcal{M}_{i}\right)$, under the map

$$
\bar{\partial}_{i}: \mathbb{V}_{i} \rightarrow e^{r, \ell} .
$$

Similarly, $\mathcal{Z}\left(\mathscr{K}\left(\mathcal{M}_{i}\right), g(\mathfrak{S})\right)$ is given by the preimage of $g\left(s_{r}\right)$. Since $\bar{\partial}_{i}\left(\partial \mathbb{V}_{i}\right)$ does not contain 0 , and $\mathfrak{S}$ and $g(\mathfrak{S})$ are sufficiently close to 0 , the two preimages are cobordant.

The main point of the proof is to homotop $\mathfrak{S}$ to $g(\mathfrak{S})$ near $\partial \mathcal{M}_{i}$ (i.e., for curves in $\mathbb{V}_{i}$ that are close to breaking) when it is nonempty. In order to simplify the cumbersome notation, let us assume without loss of generality that:

(**) $\mathcal{M}_{i_{j}}=g\left(\mathcal{M}_{i_{j}}\right)$ for all $i_{j}$ that appears in $I=\left(i_{1}, \ldots, i_{m}\right), m \geq 2$, such that $c(I)=(i)$ and for all $g \in G$; in particular $\mathcal{M}_{i}$ is $G$-invariant.

Step 1. Given $I=\left(i_{1}, \ldots, i_{m}\right)$ such that $c(I)=(i)$, consider the composition

$$
\mathbb{V}_{i_{1}} \times \cdots \times \mathbb{V}_{i_{m}} \times(R, \infty)^{m-1} \stackrel{\mathfrak{S}_{\left(i_{1}, \ldots, i_{m}\right)}}{\longrightarrow} \mathbb{V}_{I} \stackrel{\bar{\partial}_{I}}{\longrightarrow} e^{r_{1}, \ell} \oplus \cdots \oplus e^{r_{m}, \ell},
$$

where $r_{j}=\mathbf{t}\left(\mathcal{M}_{i_{j}}\right)$ and in particular $r_{m}=r$. As $R \rightarrow \infty$, its image approaches the image of the product map

$$
\left(\bar{\partial}_{i_{1}}, \ldots, \bar{\partial}_{i_{m}}\right): \mathbb{V}_{i_{1}} \times \cdots \times \mathbb{V}_{i_{m}} \rightarrow e^{r_{1}, \ell} \oplus \cdots \oplus e^{r_{m}, \ell} .
$$

This implies that $\operatorname{Im}\left(\bar{\partial}_{I} \circ \mathfrak{S}_{\left(i_{1}, \ldots, i_{m}\right)}\right)$ is effectively $\operatorname{Im}\left(\bar{\partial}_{i_{1}}, \ldots, \bar{\partial}_{i_{m}}\right)$. We assume that the generic point $\left(s_{r_{1}}, \ldots, s_{r_{m}}\right) \in e^{r_{1}, \ell} \oplus \cdots \oplus e^{r_{m}, \ell}$ has been chosen to avoid $\operatorname{Im}\left(\bar{\partial}_{i_{1}}, \ldots, \bar{\partial}_{i_{m}}\right)$. Note that under our assumption $\operatorname{vdim} \mathcal{M}_{i}=0$, we have

$$
(m-1)+\sum_{j=1}^{m} \operatorname{dim} \mathbb{V}_{i_{j}}=\operatorname{dim} \mathbb{V}_{I}=\sum_{j=1}^{m} \operatorname{dim} e^{r_{j}, \ell} .
$$

Remark 4.1.3. We will see that $\mathcal{Z}\left(\mathscr{K}\left(\mathcal{M}_{i}\right), \mathfrak{S}\right)$ and $\mathcal{Z}\left(\mathscr{K}\left(\mathcal{M}_{i}\right), g(\mathfrak{S})\right)$ are empty sets "near the boundary" unless $m=2$ and $\left(\operatorname{vdim} \mathcal{M}_{i_{1}}, \operatorname{vdim} \mathcal{M}_{i_{2}}\right)=(-1,0)$ or $(0,-1)$.

We now continue the proof in steps based on the value of $m$.

Step 2. Suppose that $m=2$.

Step $2 A$. Suppose that $\left(\operatorname{vdim} \mathcal{M}_{i_{1}}, \operatorname{vdim} \mathcal{M}_{i_{2}}\right)=(0,-1)$ or $(-1,0)$. We treat the former; the latter is analogous. Consider the $G$-equivariant, codimension one map

$$
\bar{\partial}_{i_{2}}: \mathbb{V}_{i_{2}} \rightarrow e^{r_{2}, \ell}
$$

Let $S_{\rho_{2}, r_{2}, \ell} \subset e^{r_{2}, \ell}$ (resp. $B_{\rho_{2}}^{r_{2}, \ell} \subset e^{r_{2}, \ell}$ ) be a sphere (resp. an open ball) of radius $0<\rho_{2} \ll \operatorname{dist}\left(\{0\}, \bar{\partial}_{i_{2}}\left(\partial \mathbb{V}_{i_{2}}\right)\right)$. The action $G \rightarrow \operatorname{GL}\left(e^{r_{2}, \ell}\right)$ factors through the orthogonal group and hence $G$ acts on $S_{\rho_{2}}^{r_{2}, \ell-1}$.

Lemma 4.1.4. If $s_{r_{2}} \in e^{r_{2}, \ell}$ is a point such that $0<\left|s_{r_{2}}\right|<\rho_{2}$ and $s_{r_{2}} \notin \operatorname{Im}\left(\bar{\partial}_{i_{2}}\right)$, then for any path $\gamma_{r_{2}}:[0,1] \rightarrow B_{\rho_{2}}^{r_{2}, \ell}$ from $s_{r_{2}}$ to $g\left(s_{r_{2}}\right)$, the signed intersection number $\left\langle\gamma_{r_{2}}, \bar{\partial}_{i_{2}}\right\rangle$ between $\gamma_{r_{2}}$ and $\bar{\partial}_{i_{2}}$ is zero. 


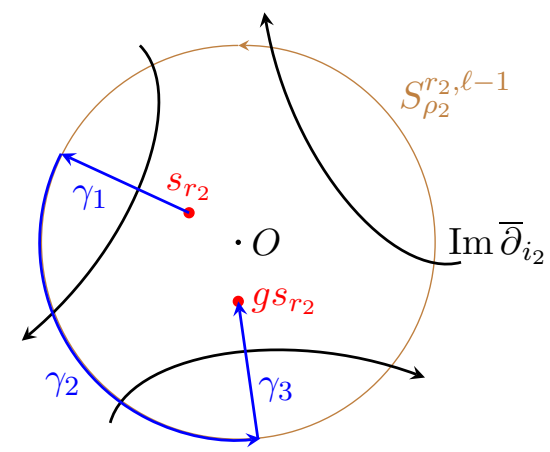

FIGURE 3.

Proof of Lemma 4.1.4 We may slightly perturb $\rho_{2}$ such that $S_{\rho_{2}}^{r_{2}, \ell-1} \pitchfork \bar{\partial}_{i_{2}}$. Then $N:=\bar{\partial}_{i_{2}}^{-1}\left(S_{\rho_{2}}^{r_{2}, \ell-1}\right)$ is a submanifold of $\mathbb{V}_{i_{2}}$ of dimension $(\ell-2)$. We homotop $\gamma_{r_{2}}$ to a concatenation $\gamma_{1} \gamma_{2} \gamma_{3}$, where

(1) $\gamma_{1}$ is a slightly perturbed radial ray from $s_{r_{2}}$ to a point $x_{1} \in C:=S_{\rho_{2}}^{r_{2}, \ell-1}-$ $\bar{\partial}_{i_{2}} N$

(2) $\gamma_{2}$ connects $x_{1}$ to $g\left(x_{1}\right)$ on $S_{\rho_{2}}^{r_{2}, \ell-1}$; and

(3) $\gamma_{3}=\left(g\left(\gamma_{1}\right)\right)^{-1}$ from $g\left(x_{1}\right)$ to $g\left(s_{r_{2}}\right)$.

See Figure 3, The contributions to $\gamma_{r_{2}} \cap \bar{\partial}_{i_{2}}$ from $\gamma_{1}$ and $\gamma_{3}$ cancel, and it remains to calculate the contribution from $\gamma_{2}$.

There exists a locally constant weight function $w: C \rightarrow \mathbb{Z}$, such that the values on adjacent connected components differ by 1 ; more precisely, given any two points $x, x^{\prime} \in C$, if $\delta$ is a path from $x$ to $x^{\prime}$ in $S_{\rho_{2}}^{r_{2}, \ell-1}$ and $\delta$ intersects $\left.\bar{\partial}_{i_{2}}\right|_{N}$ positively and only once, then $w(x)-w\left(x^{\prime}\right)=1$. The existence of such a function follows from the existence of the winding number of the map

$$
\left.\bar{\partial}_{i_{2}}\right|_{N}: N \rightarrow S_{\rho_{2}}^{r_{2}, \ell-1}-\{z\} \simeq \mathbb{R}^{\ell-1}
$$

for any $z \in C$. More precisely, for any $x \in \mathbb{R}^{\ell-1} \backslash \bar{\partial}_{i_{2}}(N), w(x)$ is given by the degree of the mapping from $N$ to $\mathbb{R}^{\ell-1} \backslash\{x\} \cong S^{\ell-2}$. Any two weight functions differ by an integer-valued constant function (depending on the choice of $z$ ).

Next we claim that $w=w \circ g$ for any $g \in G$. First observe that $w \circ g$ is also a weight function. Arguing by contradiction, suppose there is a component $C_{0}$ of $C$ such that $w\left(g\left(C_{0}\right)\right)=w\left(C_{0}\right)+k, k \neq 0$. Then $w \circ g=w+k$, and $w\left(g^{2}\left(C_{0}\right)\right)=(w \circ g)\left(C_{0}\right)+k=w\left(C_{0}\right)+2 k$. Applying this procedure to the order $m$ of the group $G, w\left(C_{0}\right)=w\left(g^{m}\left(C_{0}\right)\right)=w\left(C_{0}\right)+m k$, which is a contradiction.

Since $\left\langle\gamma_{2}, \bar{\partial}_{i_{2}}\right\rangle=\left\langle\gamma_{2},\left.\bar{\partial}_{i_{2}}\right|_{N}\right\rangle^{\circ}$, where $\langle\cdot, \cdot\rangle^{\circ}$ is the intersection number on $S_{\rho_{2}}^{r_{2}, \ell-1}$, and

$$
\left\langle\gamma_{2},\left.\bar{\partial}_{i_{2}}\right|_{N}\right\rangle^{\circ}=w\left(x_{1}\right)-w\left(g\left(x_{1}\right)\right)=0
$$

the lemma follows. 


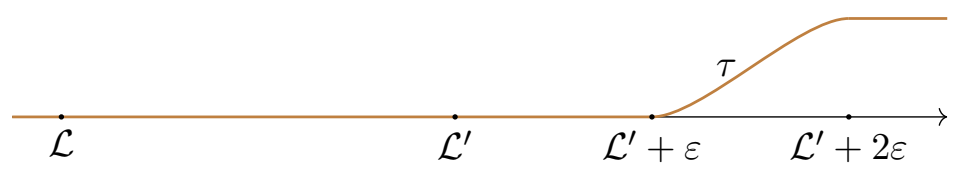

FIGURE 4.

We now explain how to homotop the section $\mathfrak{S}$ "near the boundary of" $\mathbb{V}_{i}$ to another section $\mathfrak{S}^{\prime}$ such that:

(M1) $\mathfrak{S}^{\prime}$ and $g(\mathfrak{S})$ agree "near the boundary"; and

(M2) $\mathfrak{S}$ and $\mathfrak{S}^{\prime}$ have the same signed count of intersections with $\bar{\partial}$.

In the $m=2$ case, this means that we homotop $\mathfrak{s}_{\left(i_{1}, i_{2}\right)}$ to another section $\mathfrak{s}_{\left(i_{1}, i_{2}\right)}^{\prime}$ such that:

(1) $\mathfrak{s}_{\left(i_{1}, i_{2}\right)}^{\prime}$ and $g\left(\mathfrak{s}_{\left(i_{1}, i_{2}\right)}\right)$ agree "near the boundary"; and

(2) $\mathfrak{s}_{\left(i_{1}, i_{2}\right)}$ and $\mathfrak{s}_{\left(i_{1}, i_{2}\right)}^{\prime}$ have the same signed count of intersections with $\bar{\partial}_{\left(i_{1}, i_{2}\right)}$.

Pick $\mathcal{L}^{\prime} \gg \mathcal{L}$ and let $\tau:\left[\mathcal{L}^{\prime}, \infty\right) \rightarrow[0,1]$ be a smooth function such that

- $\tau\left(\left[\mathcal{L}^{\prime}, \mathcal{L}^{\prime}+\varepsilon^{\prime \prime}\right]\right)=0$,

- $\tau\left(\left[\mathcal{L}^{\prime}+2 \varepsilon^{\prime \prime}, \infty\right)\right)=1$, and

- its restriction to $\left(\mathcal{L}^{\prime}+\varepsilon^{\prime \prime}, \mathcal{L}^{\prime}+2 \varepsilon^{\prime \prime}\right)$ is a diffeomorphism onto $(0,1)$.

Let $\gamma_{r_{j}}^{*}=\gamma_{r_{j}} \circ \tau$, where we take $\gamma_{r_{1}}$ to be an arbitrary path in $e^{r_{1}, \ell}$ connecting $s_{r_{1}}$ to $g\left(s_{r_{1}}\right)$ and $\gamma_{r_{2}}$ to be as in Lemma 4.1.4. We then define

$$
\mathfrak{s}_{\left(i_{1}, i_{2}\right)}^{\prime}=\left\{\begin{array}{cl}
\left(\gamma_{r_{1}}^{*}\left(\mathfrak{n l}_{i_{1}}\right), \gamma_{r_{2}}^{*}\left(\mathfrak{n l}_{i_{1}}\right)\right), & \text { for } \mathfrak{n l}_{i_{1}} \geq \mathcal{L}^{\prime} \\
\mathfrak{s}_{\left(i_{1}, i_{2}\right)}, & \text { for } \mathfrak{n l}_{i_{1}} \leq \mathcal{L}^{\prime}
\end{array}\right.
$$

By Lemma 4.1.4, $\mathfrak{S}$ and $\mathfrak{S}^{\prime}$ have the same signed count of intersections with $\bar{\partial}$ near $\mathbb{V}_{\left(i_{1}, i_{2}\right)}$.

Step $2 B$. Suppose that $\left(\operatorname{vdim} \mathcal{M}_{i_{1}}, \operatorname{vdim} \mathcal{M}_{i_{2}}\right)=(a,-a-1)$ or $(-a-1, a)$ with $a>0$; we treat the former. By Equation (4.1.1), a generic path $\gamma_{r_{2}}$ from $s_{r_{2}}$ to $g\left(s_{r_{2}}\right)$ does not intersect $\bar{\partial}_{i_{2}}$ and the same construction of $\mathfrak{S}^{\prime}$ applies. This covers the homotopy of $\mathfrak{S}$ near $\mathbb{V}_{I}$ for $m=2$.

Step 3. Suppose $m=3$. Let $r_{j}=\mathbf{t}\left(\mathcal{M}_{i_{j}}\right), j=1,2,3$.

Step 3A. Suppose that $\operatorname{vim} \mathcal{M}_{c\left(i_{2}, i_{3}\right)} \leq-1$.

Now we have the following variant of Lemma 4.1.4.

Lemma 4.1.5. There exists $\rho_{3}>0$ small such that if $s_{r_{3}} \in e^{r_{3}, \ell}$ is a point such that $0<\left|s_{r_{3}}\right|<\rho_{3}$ and $s_{r_{3}} \notin \operatorname{Im}\left(\bar{\partial}_{c\left(i_{2}, i_{3}\right)}\right)$, then there exists a path $\gamma_{r_{3}}:[0,1] \rightarrow B_{\rho_{3}}^{r_{3}, \ell}$ from $s_{r_{3}}$ to $g\left(s_{r_{3}}\right)$ such that:

(1) the signed intersection number $\left\langle\gamma_{r_{3}}, \bar{\partial}_{c\left(i_{2}, i_{3}\right)}\right\rangle$ is zero and

(2) $\gamma_{r_{3}}$ is disjoint from $\bar{\partial}_{c\left(i_{2}, i_{3}\right)}\left(\partial \mathbb{V}_{c\left(i_{2}, i_{3}\right)}\right)$.

Proof of Lemma 4.1.5 
Case vdim $\mathcal{M}_{c\left(i_{2}, i_{3}\right)}=-1$. In this case the proof follows the same outline as that of Lemma 4.1.4, but

$$
N:=\bar{\partial}_{c\left(i_{2}, i_{3}\right)}^{-1}\left(S_{\rho_{3}}^{r_{3}, \ell-1}\right)
$$

is now a manifold with boundary. Let us write $N=N^{\prime} \cup N^{\prime \prime}$, where $N^{\prime}$ is closed and each component of $N^{\prime \prime}$ has nonempty boundary. Writing $\gamma_{r_{3}}$ as $\gamma_{1} \gamma_{2} \gamma_{3}$ as before,

$$
\left\langle\gamma_{2}, \bar{\partial}_{c\left(i_{2}, i_{3}\right)}\right\rangle=\left\langle\gamma_{2},\left.\bar{\partial}_{c\left(i_{2}, i_{3}\right)}\right|_{N}\right\rangle^{\circ}=\left\langle\gamma_{2},\left.\bar{\partial}_{c\left(i_{2}, i_{3}\right)}\right|_{N^{\prime}}+\left.\bar{\partial}_{c\left(i_{2}, i_{3}\right)}\right|_{N^{\prime \prime}}\right\rangle^{\circ},
$$

where $\langle\cdot, \cdot\rangle^{\circ}$ is the intersection number on $S_{\rho_{3}}^{r_{3}, \ell-1}$. As before, $\left\langle\gamma_{2},\left.\bar{\partial}_{c\left(i_{2}, i_{3}\right)}\right|_{N^{\prime}}\right\rangle^{\circ}=$ 0 . We can modify $\gamma_{2}$ if $\left\langle\gamma_{2},\left.\bar{\partial}_{c\left(i_{2}, i_{3}\right)}\right|_{N^{\prime \prime}}\right\rangle^{\circ}=k$ by concatenating it with a loop in $S_{\rho_{3}}^{r_{3}, \ell-1}$ that winds $-k$ times around $\left.\bar{\partial}_{c\left(i_{2}, i_{3}\right)}\right|_{\partial N^{\prime \prime}}$. The resulting $\gamma_{2}$ will have zero signed intersection with $\left.\bar{\partial}_{c\left(i_{2}, i_{3}\right)}\right|_{N^{\prime \prime}}$, implying (1). (2) is immediate since $\left.\bar{\partial}_{c\left(i_{2}, i_{3}\right)}\right|_{\partial \mathbb{V}_{c\left(i_{2}, i_{3}\right)}}$ is a codimension two map.

Case vdim $\mathcal{M}_{c\left(i_{2}, i_{3}\right)}<-1$. In this case $\gamma_{r_{3}}$ can just be a generic arc from $s_{r_{3}}$ to $g\left(s_{r_{3}}\right)$ and it will have no intersections with $\bar{\partial}_{c\left(i_{2}, i_{3}\right)}$.

We now explain how to modify $\mathfrak{S}$ to $\mathfrak{S}^{\prime}$ near the codimension one and two "boundaries" of $\mathbb{V}_{i}$ so that (M1) and (M2) hold. In other words, we modify the sections

$$
\left(\mathfrak{s}_{\left(i_{1}, i_{2}, i_{3}\right)}, \mathfrak{s}_{\left(i_{1}, c\left(i_{2}, i_{3}\right)\right)}, \mathfrak{s}_{\left(c\left(i_{1}, i_{2}\right), i_{3}\right)}, \mathfrak{s}_{c\left(i_{1}, i_{2}, i_{3}\right)}\right)
$$

to

$$
\left(\mathfrak{s}_{\left(i_{1}, i_{2}, i_{3}\right)}^{\prime}, \mathfrak{s}_{\left(i_{1}, c\left(i_{2}, i_{3}\right)\right)}^{\prime}, \mathfrak{s}_{\left(c\left(i_{1}, i_{2}\right), i_{3}\right)}^{\prime}, \mathfrak{s}_{c\left(i_{1}, i_{2}, i_{3}\right)}\right) .
$$

The modifications will take place on the set

$$
X=\left\{\mathfrak{n l}_{i_{1}} \geq \mathcal{L}^{\prime}\right\} \cup\left\{\mathfrak{n l}_{i_{2}} \geq \mathcal{L}^{\prime}\right\},
$$

where $\mathcal{L}^{\prime} \gg \mathcal{L}$; in other words, $\mathfrak{s}_{*}=\mathfrak{s}_{*}^{\prime}$ on the complement of $X$. In the rest of this step we encourage the reader to refer to Figure 2 for the picture of a corner, where $i_{1}, i_{2}, i_{3}, c\left(i_{1}, i_{2}\right), c\left(i_{2}, i_{3}\right), c\left(i_{1}, i_{2}, i_{3}\right)$ are labeled $1-6$.

First we define

$$
\mathfrak{s}_{\left(i_{1}, c\left(i_{2}, i_{3}\right)\right)}^{\prime}=\left\{\begin{array}{cl}
\left(\gamma_{r_{1}}^{*}\left(\mathfrak{n l}_{i_{1}}\right), \gamma_{r_{3}}^{*}\left(\mathfrak{n l}_{i_{1}}\right)\right), & \text { for } \mathfrak{n l}_{i_{1}} \geq \mathcal{L}^{\prime}, \\
\mathfrak{s}_{\left(i_{1}, c\left(i_{2}, i_{3}\right)\right)}, & \text { for } \mathfrak{n l}_{i_{1}} \leq \mathcal{L}^{\prime} .
\end{array}\right.
$$

By Lemma4.1.5 $(1)$, the signed intersection number between $\bar{\partial}_{\left(i_{1}, c\left(i_{2}, i_{3}\right)\right)}$ and $\mathfrak{s}_{\left(i_{1}, c\left(i_{2}, i_{3}\right)\right)}$ on $\mathfrak{n l}_{i_{1}} \geq \mathcal{L}^{\prime}$ is zero.

Next consider the pushforwards of $\mathfrak{s}_{\left(i_{1}, c\left(i_{2}, i_{3}\right)\right)}$ and $\mathfrak{s}_{\left(i_{1}, c\left(i_{2}, i_{3}\right)\right)}$ under the morphism $\phi_{\left(i_{1}, c\left(i_{2}, i_{3}\right)\right),\left(i_{1}, i_{2}, i_{3}\right)}$. On the overlap

$$
X_{3,0}:=\left\{\mathfrak{n l}_{i_{1}} \geq \mathcal{L}^{\prime}, \mathcal{L}-\varepsilon^{\prime \prime} \leq \mathfrak{n l}_{i_{2}} \leq \mathcal{L}\right\}
$$

the section $\mathfrak{s}_{\left(i_{1}, c\left(i_{2}, i_{3}\right)\right)}=\left(s_{r_{1}}, s_{r_{3}}\right)$ is sent to $\mathfrak{s}_{\left(i_{1}, i_{2}, i_{3}\right)}=\left(s_{r_{1}}, 0, s_{r_{3}}\right)$ and the section $\mathfrak{s}_{\left(i_{1}, c\left(i_{2}, i_{3}\right)\right)}^{\prime}=\left(\gamma_{r_{1}}^{*}\left(\mathfrak{n l}_{i_{1}}\right), \gamma_{r_{3}}^{*}\left(\mathfrak{n l}_{i_{1}}\right)\right)$ is sent to $\mathfrak{s}_{\left(i_{1}, i_{2}, i_{3}\right)}^{\prime}=\left(\gamma_{r_{1}}^{*}\left(\mathfrak{n l}_{i_{1}}\right), 0, \gamma_{r_{3}}^{*}\left(\mathfrak{n l}_{i_{1}}\right)\right)$. By applying Lemma4.1.5 (2) to the term $\gamma_{r_{3}}^{*}\left(\mathfrak{n l}_{i_{1}}\right)$, we see that $\bar{\partial}_{\left(i_{1}, i_{3}, i_{3}\right)}$ has no intersections with $\mathfrak{s}_{\left(i_{1}, i_{2}, i_{3}\right)}^{\prime}$ on $X_{3,0}$ if we take $\varepsilon^{\prime \prime}>0$ to be sufficiently small. 
On

$$
\begin{gathered}
X_{3,1}:=\left\{\mathfrak{n l}_{i_{1}} \geq \mathcal{L}^{\prime}, \mathcal{L} \leq \mathfrak{n l}_{i_{2}} \leq \mathcal{L}+\varepsilon^{\prime \prime}\right\} \\
\mathfrak{s}_{\left(i_{1}, i_{2}, i_{3}\right)}=\left(\zeta\left(\mathfrak{n l}_{i_{1}}\right) s_{r_{1}}, \zeta\left(\mathfrak{n l}_{i_{2}}\right) s_{r_{2}}, s_{r_{3}}\right)=\left(s_{r_{1}}, \zeta\left(\mathfrak{n l}_{i_{2}}\right) s_{r_{2}}, s_{r_{3}}\right) .
\end{gathered}
$$

We then set

$$
\mathfrak{s}_{\left(i_{1}, i_{2}, i_{3}\right)}^{\prime}=\left(\gamma_{r_{1}}^{*}\left(\mathfrak{n l}_{i_{1}}\right), \zeta\left(\mathfrak{n l}_{i_{2}}\right) \gamma_{r_{2}}^{*}\left(\mathfrak{n l}_{i_{1}}\right), \gamma_{r_{3}}^{*}\left(\mathfrak{n l}_{i_{1}}\right)\right),
$$

where $\gamma_{r_{2}}:[0,1] \rightarrow B_{\rho_{2}}^{r_{2}, \ell}$ is some path from $s_{r_{2}}$ to $g\left(s_{r_{2}}\right)$ with $\rho_{2}=2\left|s_{r_{2}}\right|$.

Now we come to the key point: $\mathfrak{s}_{\left(i_{1}, i_{2}, i_{3}\right)}$ and $\mathfrak{s}_{\left(i_{1}, i_{2}, i_{3}\right)}^{\prime}$ do not intersect $\bar{\partial}_{\left(i_{1}, i_{2}, i_{3}\right)}$ on $X_{3,1}$ for $\varepsilon^{\prime \prime}>0$ sufficiently small. This is due to $\left|s_{r_{2}}\right| \ll\left|s_{r_{3}}\right|$ by Condition $*$ * Since $\gamma_{r_{3}}$ does not intersect $\bar{\partial}_{c\left(i_{2}, i_{3}\right)}\left(\partial \mathbb{V}_{c\left(i_{2}, i_{3}\right)}\right)$ by Lemma 4.1.5 $(2),\left.\bar{\partial}_{\left(i_{1}, i_{2}, i_{3}\right)}\right|_{X_{3,1}}$ does not intersect a small neighborhood of $\left(\gamma_{r_{1}}^{*}\left(\mathfrak{n l}_{i_{1}}\right), 0, \gamma_{r_{3}}^{*}\left(\mathfrak{n l}_{i_{1}}\right)\right)$. In particular, if $\left|s_{r_{2}}\right|$ is sufficiently small, then $\left.\bar{\partial}_{\left(i_{1}, i_{2}, i_{3}\right)}\right|_{X_{3,1}}$ does not intersect $\mathfrak{s}_{\left(i_{1}, i_{2}, i_{3}\right)}^{\prime} ; \mathfrak{s}_{\left(i_{1}, i_{2}, i_{3}\right)}$ is similar.

The situation for $\mathfrak{s}_{\left(c\left(i_{1}, i_{2}\right), i_{3}\right)}^{\prime}$ and $\mathfrak{s}_{\left(i_{1}, i_{2}, i_{3}\right)}^{\prime}$ on

$$
X_{0,3} \cup X_{1,3}:=\left\{\mathfrak{n l}_{i_{2}} \geq \mathcal{L}^{\prime}, \mathcal{L}-\varepsilon^{\prime \prime} \leq \mathfrak{n l}_{i_{1}} \leq \mathcal{L}+\varepsilon^{\prime \prime}\right\}
$$

is analogous (for one of Steps 3A, 3B, or 3C).

It remains to modify $\mathfrak{s}_{\left(i_{1}, i_{2}, i_{3}\right)}$ to $\mathfrak{s}_{\left(i_{1}, i_{2}, i_{3}\right)}^{\prime}$ on $X_{3,2} \cup X_{2,3} \cup X_{3,3}$, where:

$$
\begin{aligned}
X_{3,2}:= & \left\{\mathfrak{n l}_{i_{1}} \geq \mathcal{L}^{\prime}, \mathcal{L}+\varepsilon^{\prime \prime} \leq \mathfrak{n l}_{i_{2}} \leq \mathcal{L}^{\prime}\right\}, \\
X_{2,3}:= & \left\{\mathcal{L}+\varepsilon^{\prime \prime} \leq \mathfrak{n l}_{i_{1}} \leq \mathcal{L}^{\prime}, \mathfrak{n l}_{i_{2}} \geq \mathcal{L}^{\prime}\right\}, \\
& X_{3,3}:=\left\{\mathfrak{n l}_{i_{1}}, \mathfrak{n l}_{i_{2}} \geq \mathcal{L}^{\prime}\right\} .
\end{aligned}
$$

On $\left\{\mathfrak{n l}_{i_{1}}, \mathfrak{n l}_{i_{2}} \geq \mathcal{L}+\varepsilon^{\prime \prime}\right\}$ we have $\mathfrak{s}_{\left(i_{1}, i_{2}, i_{3}\right)}=\left(s_{r_{1}}, s_{r_{2}}, s_{r_{3}}\right)$. We then define $\mathfrak{s}_{\left(i_{1}, i_{2}, i_{3}\right)}^{\prime}$ as:

(1) $\left(\gamma_{r_{1}}^{*}\left(\mathfrak{n l}_{i_{1}}\right), \gamma_{r_{2}}^{*}\left(\mathfrak{n l}_{i_{1}}\right), \gamma_{r_{3}}^{*}\left(\mathfrak{n l}_{i_{1}}\right)\right)$ on $X_{3,2}$;

(2) $\left(\gamma_{r_{1}}^{*}\left(\mathfrak{n l}_{i_{2}}\right), \gamma_{r_{2}}^{*}\left(\mathfrak{n l}_{i_{2}}\right), \gamma_{r_{3}}^{*}\left(\mathfrak{n l}_{i_{2}}\right)\right)$ on $X_{2,3}$;

(3) $\left(\gamma_{r_{1}}^{*}\left(\beta\left(\mathfrak{n l}_{i_{1}}, \mathfrak{n l}_{i_{2}}\right)\right), \gamma_{r_{2}}^{*}\left(\beta\left(\mathfrak{n l}_{i_{1}}, \mathfrak{n l}_{i_{2}}\right)\right), \gamma_{r_{3}}^{*}\left(\beta\left(\mathfrak{n l}_{i_{1}}, \mathfrak{n l}_{i_{2}}\right)\right)\right)$ on $X_{3,3}$, where

$$
\beta(a, b)=\sqrt{\left(a-\mathcal{L}^{\prime}\right)^{2}+\left(b-\mathcal{L}^{\prime}\right)^{2}}-\mathcal{L}^{\prime} .
$$

The images of the maps (1)-(3) are 1-dimensional, since each is a postcomposition by $\left(\gamma_{r_{1}}^{*}, \gamma_{r_{2}}^{*}, \gamma_{r_{2}}^{*}\right)$, which has 1-dimensional image. On the other hand, by Equation (4.1.1), two of the three maps $\bar{\partial}_{i_{j}}: \mathbb{V}_{i_{j}} \rightarrow e^{r_{j}, \ell}, j=1,2,3$, have codimension at least one or one of the maps has codimension at least two. Hence if $\gamma_{r_{j}}$, $j=1,2,3$, are sufficiently generic, then $\mathfrak{s}_{\left(i_{1}, i_{2}, i_{3}\right)}$ and $\mathfrak{s}_{\left(i_{1}, i_{2}, i_{3}\right)}^{\prime}$ have no intersections with $\bar{\partial}_{\left(i_{1}, i_{2}, i_{3}\right)}$ on $X_{3,2} \cup X_{2,3} \cup X_{3,3}$.

Step 3B. Suppose that $\operatorname{vdim} \mathcal{M}_{c\left(i_{2}, i_{3}\right)}=0$. Then $\operatorname{vdim} \mathcal{M}_{i_{1}}=-1$. The only differences with Step 3A are that, assuming genericity of $\gamma_{r_{1}}$ and $\gamma_{r_{3}}$ :

- $\gamma_{r_{1}}:[0,1] \rightarrow e^{r_{1}, \ell}$ satisfies the conditions of Lemma 4.1.4 (where we replace $i_{2}$ by $i_{1}$ ) and intersects $\bar{\partial}_{i_{1}}$ at isolated points;

- $\gamma_{r_{3}}:[0,1] \rightarrow e^{r_{3}, \ell}$ intersects $\left.\bar{\partial}_{c\left(i_{2}, i_{3}\right)}\right|_{\partial \mathbb{V}_{c\left(i_{2}, i_{3}\right)}}$ at isolated points since it is a codimension one map; and 
- the intersection points do not occur at the same time in $[0,1]$.

It implies that if $\left|s_{r_{2}}\right| \ll\left|s_{r_{3}}\right|$, then $\mathfrak{s}_{\left(i_{1}, i_{2}, i_{3}\right)}$ and $\mathfrak{s}_{\left(i_{1}, i_{2}, i_{3}\right)}^{\prime}$, given by Equations (4.1.2) and (4.1.3), have no intersections with $\bar{\partial}_{\left(i_{1}, i_{2}, i_{3}\right)}$ on $X_{3,0} \cup X_{3,1}$.

Step $3 C$. Suppose that $\operatorname{vdim} \mathcal{M}_{c\left(i_{2}, i_{3}\right)} \geq 1$. Then $\operatorname{vdim} \mathcal{M}_{i_{1}} \leq-2$ and

- $\gamma_{r_{1}}:[0,1] \rightarrow e^{r_{1}, \ell}$ does not intersect $\operatorname{Im} \bar{\partial}_{i_{1}}$.

If $\left|s_{r_{2}}\right| \ll\left|s_{r_{3}}\right|$, then $\mathfrak{s}_{\left(i_{1}, i_{2}, i_{3}\right)}$ and $\mathfrak{s}_{\left(i_{1}, i_{2}, i_{3}\right)}^{\prime}$, given by Equations (4.1.2) and (4.1.3), have no intersections with $\bar{\partial}_{\left(i_{1}, i_{2}, i_{3}\right)}$ on $X_{3,0} \cup X_{3,1}$.

This implies the theorem for $m=3$. The general case is completely analogous and is only more complicated in notation.

\section{EquiVARIANT LAGRANGIAN FLOER COHOMOLOGY}

5.1. Grading. In order to $\mathbb{Z}$-grade our equivariant Lagrangian Floer cohomology groups, we require $L_{0}$ and $L_{1}$ to be G-equivariantly graded, i.e., (G1)-(G3) to hold.

(G1) $c_{1}(M, J)=0$.

Then there exists a nowhere-vanishing section $\Omega$ of $\wedge_{\mathbb{C}}^{n}\left(T^{*} M, J\right)$. Let $\operatorname{det}_{\Omega, i}^{2}$ : $L_{i} \rightarrow S^{1}$ be the map given by

$$
\operatorname{det}_{\Omega, i}^{2}\left(p_{i}\right)=\frac{\Omega\left(X_{i, 1} \wedge \cdots \wedge X_{i, n}\right)^{2}}{\left|\Omega\left(X_{i, 1} \wedge \cdots \wedge X_{i, n}\right)\right|^{2}}
$$

where $p_{i} \in L_{i}$ and $X_{i, 1}, \ldots, X_{i, n}$ is a basis of $T_{p_{i}} L_{i}$.

(G2) There exists a function $\theta_{i}: L_{i} \rightarrow \mathbb{R}$ that lifts $\operatorname{det}_{\Omega, i}^{2}$, i.e.,

$$
e^{2 \pi \sqrt{-1} \theta_{i}\left(p_{i}\right)}=\operatorname{det}_{\Omega, i}^{2}\left(p_{i}\right) .
$$

Then for each $p \in L_{0} \cap L_{1}$, we define an integer index $\mu(p)$ by the formula

$$
\mu(p)=n+\theta_{1}-\theta_{0}-2 \angle\left(T_{p} L_{0}, T_{p} L_{1}\right) .
$$

Here $\angle\left(T_{p} L_{0}, T_{p} L_{1}\right)=\alpha_{1}+\cdots+\alpha_{n}$, where the $\alpha_{i} \in\left(0, \frac{1}{2}\right)$ are defined by choosing a unitary basis $\left\{e_{1}, \ldots, e_{n}\right\}$ of $T_{p} L_{0}$ with respect to $\omega$ and $J$ and writing

$$
T_{p} L_{1}=\mathbb{R}\left\langle e^{2 \pi \sqrt{-1} \alpha_{1}} e_{1}, \ldots, e^{2 \pi \sqrt{-1} \alpha_{n}} e_{n}\right\rangle .
$$

(G3) $\mu(g p)=\mu(p)$ for all $p \in L_{0} \cap L_{1}$ and $g \in G$.

For more details on grading, we refer the reader to [Se2] or [AB, Section 2.3].

5.2. Chain complex. Recall the Novikov ring

$$
R=\left\{\sum_{i=0}^{\infty} a_{i} T^{\lambda_{i}} \mid a_{i} \in \mathbb{Z}, \lambda_{i} \in \mathbb{R}_{\geq 0}, \lambda_{0}=0 \text { and } \lim _{i \rightarrow \infty} \lambda_{i}=\infty\right\},
$$

where $T$ is the formal parameter. We define the $\mathbb{Z}$-graded $R$-module

$$
\begin{gathered}
C F^{\bullet}\left(L_{0}, L_{1}\right)=\bigoplus_{j} C F^{j}\left(L_{0}, L_{1}\right), \\
C F^{j}\left(L_{0}, L_{1}\right)=R\left\langle p \in L_{0} \cap L_{1} \mid \mu(p)=j\right\rangle .
\end{gathered}
$$


The differential

$$
d: C F^{j}\left(L_{0}, L_{1}\right) \rightarrow C F^{j+1}\left(L_{0}, L_{1}\right)
$$

is defined on the generators by

$$
d[p]=\sum \# \mathcal{Z}(\mathscr{K}(p, q ; A), \mathfrak{S}) \cdot T^{\int_{A} \omega}[q],
$$

where the sum is over all $q \in L_{0} \cap L_{1}$ and $A \in \pi_{2}(p, q)$ subject to the condition $\operatorname{vdim}(\mathcal{M}(p, q ; A))=0$.

Lemma 5.2.1. $d^{2}=0$.

Sketch of proof. We consider the ends of the 1-manifold $\mathcal{Z}(\mathscr{K}(p, q ; A), \mathfrak{S})$ where $\operatorname{vdim}(\mathcal{M}(p, q ; A))=1$. First observe that, by codimension reasons, for any $u \in$ $\mathcal{Z}(\mathscr{K}(p, q ; A), \mathfrak{S})$, we have $\mathfrak{n l}_{r}(u) \leq \mathcal{L}+\varepsilon^{\prime \prime}$ for all but possibly one $r \in L_{0} \cap$ $L_{1}$ (cf. Equation (2.5.3) for the definition of the section $\mathfrak{s}_{I}$ and Definition 2.5.3 for the definition of $\zeta$ and note that $\left.\zeta\left(\left[\mathcal{L}+\varepsilon^{\prime \prime}, \infty\right)\right)=1\right)$. Hence the ends of $\mathcal{Z}(\mathscr{K}(p, q ; A), \mathfrak{S})$ are in bijection with

$$
\coprod_{r} \mathcal{Z}\left(\mathscr{K}\left(p, r ; A_{1}\right), \mathfrak{S}_{1}\right) \times \mathcal{Z}\left(\mathscr{K}\left(r, q ; A_{2}\right), \mathfrak{S}_{2}\right),
$$

where $\operatorname{vdim}\left(\mathcal{M}\left(p, r ; A_{1}\right)\right)=\operatorname{vdim}\left(\mathcal{M}\left(r, q ; A_{2}\right)=0\right.$ and $\mathfrak{S}_{1}$ and $\mathfrak{S}_{2}$ are compatible with $\mathfrak{S}$.

We define the usual Lagrangian Floer cohomology from $L_{0}$ to $L_{1}$ by

$$
H F^{\bullet}\left(L_{0}, L_{1}\right)=\operatorname{ker} d / \operatorname{Im} d .
$$

By Theorem 4.1.2, if $\operatorname{vdim}(\mathcal{M}(p, q ; A))=0$ and $g \in G$ takes $\mathscr{K}(p, q ; A)$ to itself,then

$$
\# \mathcal{Z}(\mathscr{K}(p, q ; A), \mathfrak{S})=\# \mathcal{Z}(\mathscr{K}(p, q ; A), g(\mathfrak{S}))
$$

Hence,

$$
\begin{aligned}
d g[p] & =\sigma(g, p) d[g p] \\
& =\sigma(g, p) \sum_{r, B} \# \mathcal{Z}(\mathscr{K}(g p, r ; B), \mathfrak{S})[r] \\
& =\sigma(g, p) \sum_{q, A} \# \mathcal{Z}(\mathscr{K}(g p, g q ; g A), \mathfrak{S})[g q] \\
& =\sigma(g, p) \sum_{q, A} \# \mathcal{Z}(\mathscr{K}(g p, g q ; g A), g \mathfrak{S})[g q] \\
& =\sigma(g, p) \sum_{q, A} \#(g(\mathcal{Z}(\mathscr{K}(p, q ; A), \mathfrak{S}))[g q] \\
& =\sigma(g, p) \sum_{q, A} \# \mathcal{Z}(\mathscr{K}(p, q ; A), \mathfrak{S}) \sigma(g, p) \sigma(g, q)[g q] \\
& =g d[p],
\end{aligned}
$$

i.e., $d$ is $R[G]$-linear. 
Let $\left(P_{\bullet}, d_{P}\right)$ be a projective resolution of $R$ over $R[G]$. We denote

$$
E^{i, j}:=\operatorname{Hom}_{R[G]}\left(P_{i}, C F^{j}\left(L_{0}, L_{1}\right)\right),
$$

where $P_{i}$ and $C F^{j}\left(L_{0}, L_{1}\right)$ are regarded as $R[G]$-modules. Let $d_{>}^{i, j}: E^{i, j} \rightarrow$ $E^{i+1, j}$ be the map induced by $d_{P}: P_{i+1} \rightarrow P_{i}$, and $d_{\wedge}^{i, j}: E^{i, j} \rightarrow E^{i, j+1}$ be the map induced by $d: C F^{j}\left(L_{0}, L_{1}\right) \rightarrow C F^{j+1}\left(L_{0}, L_{1}\right)$ multiplied by the factor $(-1)^{i}$. Then $d_{>}^{i, j}$ and $d_{\wedge}^{i, j}$ commute with the multiplication by elements in $R[G]$ and form a double complex.

The G-equivariant Lagrangian Floer cochain complex is the total complex

$$
\left(C F_{G}^{\bullet}\left(L_{0}, L_{1}\right), d_{G}\right)
$$

where

$$
C F_{G}^{k}\left(L_{0}, L_{1}\right)=\bigoplus_{i+j=k} E^{i, j},\left.\quad d_{G}\right|_{E^{i, j}}=d_{>}^{i, j}+d_{\wedge}^{i, j}
$$

The corresponding $G$-equivariant Lagrangian Floer cohomology group is:

$$
H F_{G}^{\bullet}\left(L_{0}, L_{1}\right)=\operatorname{ker} d_{G} / \operatorname{Im} d_{G}
$$

The cohomology $H^{\bullet}\left(\operatorname{Hom}_{R[G]}\left(P_{\bullet}, R\right)\right) \cong H^{\bullet}(B G)$ (taking $Y=\{$ pt $\}$ as in Section (1) is a ring whose product is the standard cup product. Similarly we can define the following $R[G]$-bilinear map:

$$
H^{\bullet}(B G) \times H F_{G}^{\bullet}\left(L_{0}, L_{1}\right) \rightarrow H F_{G}^{\bullet}\left(L_{0}, L_{1}\right),
$$

which makes $H F_{G}^{\bullet}\left(L_{0}, L_{1}\right)$ an $H^{\bullet}(B G)$-module. Indeed, it is easier to see the module structure via the definition of $H_{G}^{\bullet}\left(L_{0}, L_{1}\right)$ using the singular chain complex $C_{\bullet}(E G)$ in place of $P_{\bullet}$. More precisely, the product on the chain level is induced by the composition of the Künneth map followed by the diagnal map:

$$
\begin{array}{r}
\operatorname{Hom}_{R[G]}\left(C_{i}(E G), C F^{j}\left(L_{0}, L_{1}\right)\right) \times \operatorname{Hom}_{R[G]}\left(C_{k}(E G), R\right) \\
\stackrel{\text { Künneth map }}{\longrightarrow} \operatorname{Hom}_{R[G]}\left(C_{i+k}(E G \times E G), C F^{j}\left(L_{0}, L_{1}\right)\right) \\
\stackrel{\Delta^{*}}{\longrightarrow} \operatorname{Hom}_{R[G]}\left(C_{i+k}(E G), C F^{j}\left(L_{0}, L_{1}\right)\right) .
\end{array}
$$

From standard results on spectral sequences of double complexes, we obtain:

Lemma 5.2.2. There exists a spectral sequence $\left\{E_{r}^{i, j}\right\}_{r}$ with second page

$$
E_{2}^{i, j}=\operatorname{Ext}_{R[G]}^{i}\left(R, H F^{j}\left(L_{0}, L_{1}\right)\right)
$$

converging to $H F_{G}^{\bullet}\left(L_{0}, L_{1}\right)$.

5.3. Chain map. Using the notation from Section 2.8, for $p \in L_{0} \cap L_{1}, q \in$ $L_{0}^{\prime} \cap L_{1}^{\prime}$, and $A \in \pi_{2}(p, q)$, there exists a Kuranishi structure $\mathscr{K}(p, q ; A)$ and a collection of sections $\mathfrak{S}$ such that chain map

$$
\Phi: C F^{\bullet}\left(L_{0}, L_{1}\right) \rightarrow C F^{\bullet}\left(L_{0}^{\prime}, L_{1}^{\prime}\right)
$$

is defined on the generators by

$$
\Phi(p)=\sum \# \mathcal{Z}(\mathscr{K}(p, q ; A), \mathfrak{S}) \cdot T^{\int_{A} \omega} q,
$$


where the sum is over all $q \in L_{0}^{\prime} \cap L_{1}^{\prime}$ and $A \in \pi_{2}(p, q)$ subject to the conditions $\operatorname{vdim}\left(\mathcal{M}^{\circ}(p, q ; A)\right)=0$ and $(p, q, A)$ belongs to Sequence (2.5.2). We also have the following, whose proof is similar to that of Lemma 5.2.1

Lemma 5.3.1. $d \circ \Phi=\Phi \circ d$.

The proof of Theorem 4.1.2 carries over for chain maps. In other words, if $\operatorname{vdim}\left(\mathcal{M}^{\circ}(p, q ; A)\right)=0$ and $g \in G$ preserves $\mathscr{K}(p, q ; A)$, then

$$
\# \mathcal{Z}(\mathscr{K}(p, q ; A), \mathfrak{S})=\# \mathcal{Z}(\mathscr{K}(p, q ; A), g(\mathfrak{S})) .
$$

This implies that:

Lemma 5.3.2. $\Phi: C F^{\bullet}\left(L_{0}, L_{1}\right) \rightarrow C F^{\bullet}\left(L_{0}^{\prime}, L_{1}^{\prime}\right)$ is a chain map of $R[G]$ modules.

It is clear from the definition that the chain map $\Phi$ induces the chain map

$$
\Phi_{G}: C F_{G}^{\bullet}\left(L_{0}, L_{1}\right) \rightarrow C F_{G}^{\bullet}\left(L_{0}^{\prime}, L_{1}^{\prime}\right) .
$$

\subsection{Chain homotopy. Let}

$$
\Phi: C F^{\bullet}\left(L_{0}, L_{1}\right) \rightarrow C F^{\bullet}\left(L_{0}^{\prime}, L_{1}^{\prime}\right) \quad \text { and } \quad \Psi: C F^{\bullet}\left(L_{0}^{\prime}, L_{1}^{\prime}\right) \rightarrow C F^{\bullet}\left(L_{0}, L_{1}\right)
$$

be chain maps of $R[G]$-modules, defined using $\phi_{s}$ and $\phi_{s}^{-1}$.

Fix $p \in L_{0} \cap L_{1}, q \in L_{0}^{\prime} \cap L_{1}^{\prime}$, and $A \in \pi_{2}(p, q)$. Using the function $\Theta$ from Section 2.8.2 we construct the bundles $\pi_{I,[0,1]}: \mathbb{E}_{I,[0,1]} \rightarrow \mathbb{V}_{I,[0,1]}$ and the 1-parameter family

$$
\mathscr{K}_{[0,1]}(p, q ; A):=\coprod_{\tau \in[0,1]} \mathscr{K}_{\tau}(p, q ; A),
$$

of Kuranishi structures. Here each term $\mathscr{K}_{\tau}(p, q ; A)$ corresponds to $\mathscr{K}(p, q ; A)$ for $\tau \in[0,1]$. We can take the sections $\mathfrak{s}_{I,[0,1]}$ of $\mathfrak{S}_{[0,1]}$, viewed as a map to a fixed vector space, to be "independent of $\tau$ " or, more precisely, only dependent on neck lengths.

Now consider the ends of $\mathcal{Z}\left(\mathscr{K}_{[0,1]}(p, q ; A), \mathfrak{S}_{[0,1]}\right)$ for $\operatorname{vdim}(\mathcal{M}(p, q ; A))=0$. A similar argument as Lemma 5.3.2 gives

Lemma 5.4.1. $\Phi \circ \Psi-\mathrm{id}=K \circ d+d \circ K$, where

$$
K: C F^{\bullet}\left(L_{0}, L_{1}\right) \rightarrow C F^{\bullet}\left(L_{0}, L_{1}\right)
$$

is a map of $R[G]$-modules.

Let $K_{G}: C F_{G}^{\bullet}\left(L_{0}, L_{1}\right) \rightarrow C F_{G}^{\bullet}\left(L_{0}, L_{1}\right)$ be the map induced by $K$. We obtain

$$
\Phi_{G} \circ \Psi_{G}-\mathrm{id}=K_{G} \circ d_{G}+d_{G} \circ K_{G} .
$$

Summarizing, we have:

Corollary 5.4.2. The G-equivariant Lagrangian Floer cohomology $H F_{G}^{\bullet}\left(L_{0}, L_{1}\right)$ is independent of the choice of equivariant almost complex structure $J$ and is an invariant of the pair $\left(L_{0}, L_{1}\right)$ under $G$-equivariant Hamiltonian isotopy. 


\section{REFERENCES}

[AB] G. Alston and E. Bao, Exact, graded, immersed Lagrangians and Floer theory, J. Symplectic Geom. 16 (2018), 357-438.

[BH] E. Bao and K. Honda, Definition of cylindrical contact homology in dimension three, J. Topol. 11 (2018) 1001-1052.

[BH2] E. Bao and K. Honda, Semi-global Kuranishi charts and the definition of contact homology, preprint 2015. ArXiv:1512.00580.

$[\mathrm{CH}]$ C. Cho and H. Hong, Finite group actions on Lagrangian Floer theory, J. Symplectic Geom. 15 (2017), 307-420.

[EES] T. Ekholm, J. Etnyre, and M. Sullivan, Orientations in Legendrian contact homology and exact Lagrangian immersions, Internat. J. Math. 16 (2005), 453-532.

[ES] T. Ekholm, and I. Smith, Exact Lagrangian immersions with one double point revisited, Math. Annalen 358, no. 1-2 (2014): 195-240.

[Fu] K. Fukaya, Lie groupoid, deformation of unstable curve, and construction of equivariant Kuranishi charts. arXiv preprint arXiv:1701.02840 (2017).

[FO] K. Fukaya and Y. Oh, Zero-loop open strings in the cotangent bundle and Morse homotopy, Asian J. Math. 1 (1997), 96-180.

[FOn] K. Fukaya and K. Ono, Arnold conjecture and Gromov-Witten invariant, Topology 38 (1999), 933-1048.

[FO3] K. Fukaya, Y. Oh, H. Ohta and K. Ono, Lagrangian intersection Floer theory: anomaly and obstruction, Parts I and II, AMS/IP Studies in Advanced Mathematics, 46.1 and 46.2. American Mathematical Society, Providence, RI; International Press, Somerville, MA, 2009.

[He1] K. Hendricks, A rank inequality for the knot Floer homology of double branched covers, Algebraic \& Geom. Topol. (2012), 12(4), pp.2127-2178.

[He2] K. Hendricks, Localization and the link Floer homology of doubly-periodic knots, J. Symplectic Geom. 13 (2015), 545-608.

[He3] K. Hendricks, A spectral sequence of the Floer cohomology of symplectomorphisms of trivial polarization class, Internat. Math. Research Notices. (2017), pp.509-528.

[HLS] K. Hendricks, R. Lipshitz and S. Sarkar, A flexible construction of equivariant Floer homology and applications, J. Topol. 9 (2016), 1153-1236.

[KS] M. Khovanov and P. Seidel, Quivers, Floer cohomology, and braid group actions, J. Amer. Math. Soc. 15 (2002), 203-271.

[MS1] D. McDuff and D. Salamon, Introduction to symplectic topology. Second edition. Oxford Mathematical Monographs. The Clarendon Press, Oxford University Press, New York, 1998.

[MS2] D. McDuff and D. Salamon, J-holomorphic curves and symplectic topology, American Mathematical Society Colloquium Publications, 52. American Mathematical Society, Providence, RI, 2004.

[MW] D. McDuff and K. Wehrheim, Smooth Kuranishi atlases with trivial isotropy, Geom. Topol. 21 (2017), 2725-2809.

[Pa] J. Pardon, An algebraic approach to virtual fundamental cycles on moduli spaces of pseudoholomorphic curves, Geom. Topol. 20 (2016), 779-1034.

[Sc] F. Schmäschke, Floer homology of Lagrangians in clean intersection, preprint 2016. ArXiv: 1606.05327.

[Se] P. Seidel, Fukaya categories and Picard-Lefschetz, theory. Zurich Lectures in Advanced Mathematics. European Mathematical Society (EMS), Zürich, 2008.

[Se2] P. Seidel, Graded Lagrangian submanifolds, Bull. Soc. Math. France 128 (2000), 103-149.

[SS] P. Seidel and I. Smith, Localization for involutions in Floer cohomology, Geom. Funct. Anal. 20 (2010), 1464-1501.

[Si] A. Silva, Lectures on Symplectic Geometry, Lecture Notes in Mathematics, 1764. SpringerVerlag, Berlin, 2001.

[So] J. Solomon, Intersection theory on the moduli space of holomorphic curves with Lagrangian boundary conditions, preprint 2006. ArXiv:0606429. 
[WW] K. Wehrheim and C. Woodward, Orientations for pseudoholomorphic quilts, preprint 2015. ArXiv:1503.07803.

Simons Center for Geometry and Physics, State University of New York, Stony BROOK, NY 11790

E-mail address: baoerkao@gmail.com

University of CAlifornia, Los Angeles, Los Angeles, CA 90095

E-mail address: honda@math.ucla.edu

URL: http: //www.math.ucla.edu/ honda 